Ralf Klausnitzer

\title{
Philologen in der NS-Schrifttumspolitik und ihre Observationen der Gegenwartsliteratur 1933-1945
}

DOI 10.1515/iasl-2016-0027

\begin{abstract}
During the 1930s and 1940s, the publishing sphere in Germany had to adapt to substantial ideological imperatives. It was subject to the control of various often rivaling institutions of censorship. These censoring institutions employed literary scholars to observe and influence the growing production of books and magazines. This paper reconstructs the practices these literary scholars employed for dealing with contemporary literature in an ideologically conditioned setting. It engages with the still largely unexplored relation between academic socialization, interpretative practices and politically motivated interventions.
\end{abstract}

Die kulturelle und wirtschaftliche Bedeutung der Literatur- und Buchproduktion bleibt auch nach der Machtübernahme 1933 und den Versuchen zur ,Gleichschaltung' nicht zu unterschätzen: Sowohl hinsichtlich der jährlichen Gesamtproduktion als auch in Bezug auf die Anzahl der Neuerscheinungen nimmt das nationalsozialistische Deutschland weiterhin die Spitzenposition in Europa ein; in der Warenstatistik rangiert das Buch (nach Steinkohle und Weizen) auf dem dritten Platz. ${ }^{1}$ Politische Weichenstellungen und willfährige Anpassungsleistungen transformieren die plurale Kulturlandschaft der Weimarer Republik zwar in eine Mediendiktatur, die den Ansprüchen des autoritären Regimes verpflichtet ist doch auf eingeführte Größen des literarischen Lebens und Pluralität kann man ebenso wenig verzichten wie auf länderübergreifende Austauschbeziehungen. International erfolgreiche Werke wie Vom Winde verweht von Margaret Mitchell oder Wind, Sand und Sterne von Antoine de Saint-Exupéry, dem Autor des Kleinen Prinzen und Flieger gegen die NS-Luftwaffe, avancieren in Nazi-Deutschland mit

1 Vgl. Jan-Pieter Barbian: Literaturpolitik im NS-Staat. Von der „Gleichschaltung“ bis zum Ruin. Frankfurt/M.: Fischer Taschenbuch Verlag 2010, S. 429.

Kontaktdaten: PD Dr. Ralf Klausnitzer, Humboldt-Universität zu Berlin, Philosophische Fakultät II, Institut für deutsche Literatur, Unter den Linden 6, 10099 Berlin,

E-Mail: ralf.klausnitzer@rz.hu-berlin.de 
ca. 300.000 bzw. 135.000 verkauften Exemplaren ebenso zu Bestsellern wie die Romane von Ehm Welk oder Ernst Wiechert, deren Autoren kurzzeitig in Konzentrationslager gebracht worden waren. - Auch im Vergleich mit den modernen und kollektiv wirksamen Medien Film und Rundfunk verlieren individuell zu rezipierende Bücher keineswegs an gesellschaftlicher Relevanz und Signifikanz. Im Gegenteil: Das Buch behauptet auch in der Zeit der NS-Herrschaft seinen eminenten kulturellen und wirtschaftlichen Status, bevor die in den 1950er Jahren einsetzende Bilderflut des Fernsehens und die sich ausbreitende Populärkultur die auf Papier und Druckerschwärze gebaute Welt von Lesern nachhaltig verändern wird.

Eben deshalb entwickeln die kulturpolitischen Akteure des Regimes diverse Instanzen zur Überwachung und Reglementierung des literarischen Lebens. Die Bücher- und Zeitschriftenproduktion soll ebenso beobachtet und gesteuert werden wie die kritische und wissenschaftliche Kommunikation über Literatur. Wesentlich beteiligt an diesen Prozessen sind Institutionen, die sich in besonderer Weise auf die aktuelle Literaturproduktion und deren kritische bzw. wissenschaftliche Bearbeitung konzentrieren: Neben der schon 1933 gegründeten Reichsschrifttumskammer (RSK) und der 1934 geschaffenen Abteilung VIII (Schrifttum) im Reichsministerium für Volksaufklärung und Propaganda (RMVP) operieren in der NSDAP die Parteiamtliche Prüfungskommission unter Reichsleiter Philipp Bouhler, die für Gutachten verantwortliche Parteikanzlei und die Dienststelle zur Überwachung der gesamten politischen und weltanschaulichen Schulung unter Alfred Rosenberg, die in der Reichsstelle zur Förderung des deutschen Schrifttums und der monatlich erscheinenden Zeitschrift Bücherkunde eine wachsende Zahl haupt- und nebenamtlicher Lektoren beschäftigt. Die SS besitzt mit dem von zahlreichen Germanisten und Historikern durchsetzten Sicherheitsdienst (SD) eigene Beobachtungsinstrumente, die das erscheinende Schrifttum kontrollieren und eine weltanschaulich motivierte ,Gegnerforschung praktizieren. In allen Institutionen der NS-Schrifttumspolitik arbeiten universitär ausgebildete Geisteswissenschaftler, die Textkompetenzen und Weltanschauungsdirektiven nutzen, um die bis 1942 kontinuierlich steigende Buch- und Zeitschriften-Produktion (die trotz nachhaltiger Konzentrationsprozesse im Verlagswesen und knapper Ressourcen beträchtlich bleibt) zu observieren und zu dirigieren. Der Beitrag rekonstruiert Beobachtungspraktiken von Philologen in diesen weltanschaulich-ideologisch konditionierten Umgangsformen mit Gegenwartsliteratur und widmet sich dabei vor allem bislang ungeklärten Zusammenhängen zwischen wissenschaftlicher Sozialisation, Textbeobachtungsverfahren und politisch induzierten Eingriffen.

Die Beobachtung von Gegenwartsliteratur durch Philologen in den Schrifttumsinstanzen des NS-Regimes ist aus mehreren Gründen von Interesse. Denn die 
hier zu rekonstruierenden Verhältnisse zeigen zum einen, wie und mit welchen Verfahren aktive Produzenten von Gegenwartsliteratur im Spannungsfeld politischer Lenkungsansprüche observiert und bewertet werden - und wie Autoren, Verleger, Leser auf diese Beobachtungen reagieren. Sie dokumentieren zum anderen die Beobachtungsdispositive und Wertungskriterien von Vertretern der disziplinären Literaturwissenschaft, die als promovierte und habilitierte Geisteswissenschaftler in politischen Institutionen des NS-Staates wirken und sehr spezielle Umgangsformen mit der aktuellen Literaturproduktion entwickeln: Freiwillig und mit zum Teil beträchtlichem Elan übernehmen sie die Aufgabe, politische Steuerungsvorstellungen im Buch- und Verlagswesen des totalitären Staates durchzusetzen und ein mehrfach dimensioniertes System der Überwachung und Kontrolle $\mathrm{zu}$ bedienen wie $\mathrm{zu}$ perfektionieren. Besonders aktiv sind dabei einerseits die im SD versammelten Philologen um den Hermann-August-Korff-Schüler Wilhelm Spengler (der in der Deutschen Bücherei Leipzig eine Dienststelle zur Überwachung des eingehenden Schrifttums aufbaut) und den ebenfalls literaturwissenschaftlich promovierten Hans Rößner (der bei Karl Justus Obenauer in Bonn mit einer Arbeit George-Kreis und Literaturwissenschaft. Zur Würdigung und Kritik der geistigen Bewegung Stefan Georges den Doktortitel erworben hatte und unter anderem das berüchtigte SD-Dossier Lage und Aufgaben der Germanistik und deutschen Literaturwissenschaft anfertigt, bevor er nach dem Krieg zum Verlagsleiter des Piper-Verlags aufsteigen und dort unter anderem die Werke von Hannah Arendt betreuen sollte). Literaturwissenschaftler wirken zum anderen als haupt- und nebenamtliche Lektoren in Alfred Rosenbergs Dienststelle für die gesamte politische und weltanschauliche Schulung der NSDAP; zu ihnen gehören der Berliner Ordinarius Franz Koch sowie zahlreiche weitere Germanisten, die nach dem Zusammenbruch des Regimes ihre Karriere fortsetzen sollten. In besonderer Weise vorzustellen sind die gleichfalls philologisch ausgebildeten Mitarbeiter der 1934 gebildeten Abteilung VIII (Schrifttum) des Reichsministeriums für Volksaufklärung und Propaganda, die nachhaltige Bedeutung für Beobachtung und Indizierung der Gegenwartsliteratur gewinnen sollten. Im Mittelpunkt steht dabei der 1934 promovierte und 1944 habilitierte Literaturwissenschaftler und Goethe-Forscher Wilhelm Emrich, der zwischen Januar 1942 und März 1944 als ,Erkundungsreferent' des RMVP an der Deutschen Bücherei in Leipzig sowie im Berliner Ministerium auch mit der aktuellen Literaturproduktion befasst ist. Nach dem Zusammenbruch des Regimes als „entlastet“ entnazifiziert, macht er erfolgreich akademische Karriere, die ihn nach Stationen in Göttingen und Köln 1959 als ordentlichen Professor an die Freie Universität nach Berlin führt. Mit einflussreichen Arbeiten über Franz Kafka und Gesamtausgaben der Werke von Carl Sternheim, Arno Holz und Ricarda Huch wird er die Moderne-Forschung vorantreiben; zur Gegenwartsliteratur äußert er sich unter anderem im Rahmen 
der Universitätstage 1964 mit dem Vortrag „Kann Dichtung verboten werden?“ was angesichts seiner früheren Tätigkeit im „Verbotsreferat“ des Reichspropagandaministeriums besondere Brisanz gewinnt.

\section{Die „staatliche[] Welt des Buches“ und die Gegenwartsliteratur}

Die vielfältigen Bemühungen zur politischen Kontrolle und Reglementierung des literarisch-kulturellen sowie des wissenschaftlichen Lebens zwischen 1933 und 1945 sind an dieser Stelle nicht einmal in Umrissen nachzuzeichnen. Einen Überblick vermittelt das Organigramm „Gesamtaufbau der staatlichen Welt des Buches“, das in dem von Hellmuth Langenbucher herausgegebenen Kompendium Die Welt des Buches abgedruckt ist:

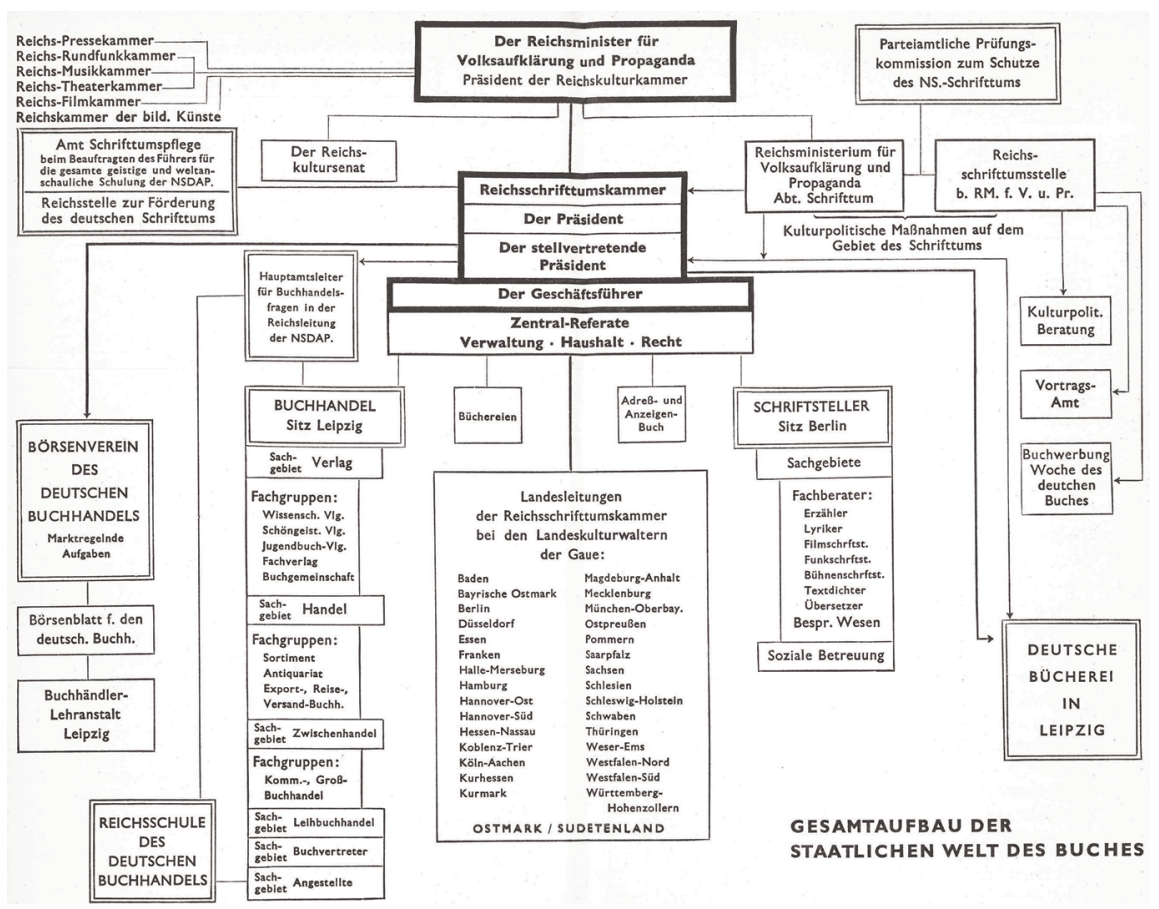

Abb. 1: Gesamtaufbau der staatlichen Welt des Buches. In: Hellmuth Langenbucher (Hg.): Die Welt des Buches. Eine Kunde vom Buch. Unter Mitarbeit von Karl Heinrich Bischoff. Mit einem Geleitwort von Hanns Johst. Ebenhausen bei München: Wilhelm Langewiesche-Brandt 1938, S. 193 f. 
Die hier schematisierten Institutionen stehen seit Beginn der NS-Herrschaft in mehrfachen Spannungsverhältnissen. Einerseits nehmen Buchproduktion und -verbreitung auch unter den Bedingungen kulturell- und wissenschaftspolitischer ,Gleichschaltung' zu und machen das Buch und die mit ihm verbundenen Vorgänge der Herstellung, Verbreitung, Bewerbung und Bewertung zu einem mehrfach dimensionierten Kultur- und Wirtschaftsfaktor. ${ }^{2}$ - Andererseits setzen im Verlagswesen und im Buchhandel signifikante Konzentrationsprozesse ein, die dazu führen, dass die Branche in diesen Jahren einen fundamentalen Strukturwandel durchmacht: 1933 gibt es in Deutschland rund 2.000 Verlage, 1945 sind es noch 200. ${ }^{3}$ Der nach 1942 signifikant abnehmenden Anzahl von Buchtiteln stehen immens steigende Auflagen gegenüber. Mit der zunehmenden Ressourcenknappheit - es fehlt an Autoren, an Papier, an Drucktechnik - korrespondieren verschärfte Zensurbestimmungen, die über Mechanismen der Papiervergabe durchgesetzt werden: Verlage müssen Titel und Auflagehöhe einzeln begründen und genehmigen lassen. Vor diesem Hintergrund gewinnen die Verkaufszahlen von Büchern besondere Signifikanz: Christian Adams Auflistung der „zehn erfolg-

2 Im Jahr 1940 gab es 22.289 Neuerscheinungen bei einer Gesamtauflagenhöhe von 242 Millionen Exemplaren, die im Folgejahr 1941 auf knapp 342 Millionen Exemplare gesteigert werden konnte; vgl. Barbian: Literaturpolitik im NS-Staat (Anm. 1), S. 429. Welche ökonomische Bedeutung die Buch- und Zeitschriftenproduktion hatte, belegen weitere Zahlen: Die rund 3.500 deutschen Verlage zahlten 1937 rund 40 Mill. RM an Honoraren, 30 Mill. RM für Papier, 80 Mill. RM für Druckarbeiten, 10 Mill. RM für Klischees und 40 Mill. RM für Buchbindearbeiten. Die Buchbranche speiste damit rund 200 Mill. RM in die deutsche Volkswirtschaft ein. Die produzierten Bücher hatten einen Verkaufswert von insgesamt 650 Mill. RM und sicherten dem Buch in der Warenstatistik nach Steinkohle und Weizen den dritten Rang. Im deutschen Einzelhandel erzielten die rund 10.000 Sortimentsbuchhandlungen und der in Leipzig konzentrierte Zwischenbuchhandel einen Jahresumsatz von 483 Mill. RM - was den Verkauf von Büchern nach Zigaretten, Frauenund Mädchenbekleidung zum drittstärksten Wirtschaftsfaktor machte. Das Buchgeschäft reichte zugleich über die Reichsgrenzen hinaus: Vor dem Krieg wurden jährlich Bücher im Wert von mehr als 22 Mill. RM ausgeführt, während die Büchereinfuhr im Wert von 7,698 Mill. RM deutlich darunter lag. Vgl. Barbian: Literaturpolitik im NS-Staat (Anm. 1), S. 21 f. Vergegenwärtigt man sich zudem die Dichte von Bibliothekseinrichtungen im Deutschen Reich (zu den Staats-, Landes- und Hochschulbibliotheken kamen rund 9500 Stadt- und Volksbüchereien in kommunaler Trägerschaft), dann werden die Dimensionen der hier verhandelten Verhältnisse deutlicher.

3 Klaus G. Saur (Hg.): Verlage im „Dritten Reich“. Frankfurt/M.: Klostermann 2013. Die hier entwickelte Typologie sortiert Verlage im ,Dritten Reich“ in drei Gruppen: (a) Verlage, die verboten oder enteignet wurden bzw. von emigrierenden Verlegern im Ausland weitergeführte Verlage wie S. Fischer oder Paul Zsolnay, (b) parteinahe bzw. im Parteibesitz befindliche Verlage wie Franz Eher Nachf. oder Hanseatische Verlagsanstalt, (c) sich arrangierende oder distanzierende Verlage wie der unter Herbert Cram arbeitende Verlag Walter de Gruyter. Diese Verlage verändern partiell wissenschaftliche Schwerpunkte, meiden jüdische Autoren, bedienen die durch den Krieg entstehenden Märkte. 
reichsten Buchtypen im Dritten Reich“ verzeichnet nicht nur den beispiellosen Erfolg von ,Rohstoffromanen` wie beispielsweise Anilin von Karl Alois Schenzinger, der erstmals 1937 erscheint und bis Kriegsende in 920.000 Exemplaren verkauft wird, bevor er im Mai 1951 die Marke von 1,6 Millionen überschreitet. ${ }^{4}$ Auch zahlreiche weitere Bestseller werden nach dem Ende der NS-Herrschaft gut verkauft - von Ehm Welks Roman Die Heiden von Kummerow (der mit 730.000 verkauften Exemplaren auf Rang drei der bestverkauften Romane im Dritten Reich gelangt) bis zum Ratgeber Die deutsche Mutter und ihr erstes Kind von Johanna Haarer, der erstmals 1934 im rassenkundlich ausgewiesenen J. F. Lehmanns Verlag erscheint, es bis Kriegsende auf eine halbe Million verkaufte Exemplare bringt und (vom Attribut ,deutsch' und anderen zeittypischen Elementen befreit) bis 1987 in 1,2 Millionen Exemplaren über den Ladentisch geht. ${ }^{5}$

In diesen Prozessen spielen die diversen und sich zum Teil überschneidenden Einsätze unterschiedlicher Instanzen zur Beobachtung und Reglementierung der Buch- und Zeitschriftenproduktion eine wichtige Rolle. Ihre Aktivitäten, die vor allem von den materialgesättigten Arbeiten von Jan-Pieter Barbian dargestellt wurden, sollen hier nicht noch einmal nachgezeichnet werden. ${ }^{6}$ Hinzuweisen ist jedoch auf die Koexistenz verschiedener Institutionen, in denen vor allem Absolventen historisch-philologischer Fächer die aktuelle Literaturproduktion beobachten. Als Abteilung des ,Amtes Rosenberg“ erfüllt etwa die ,Reichsstelle zur Förderung des deutschen Schrifttums“ die Aufgabe, „das wesentliche deutschsprachige Schrifttum der neueren Zeit nach weltanschaulichen, politischen, künstlerischen und volkserzieherischen Gesichtspunkten zu begutachten“; gleichwohl verfügt man nicht über exekutive Verbotsrechte und operiert vor allem mit Mitteilungen über besonders vorbildliche und förderungswürdige Werke. ${ }^{7}$ Realisiert werden diese evaluierenden Funktionen durch haupt- und nebenamtliche Lektoren, deren Gutachten in der monatlichen Zeitschrift Bücherkunde

4 Christian Adam: Lesen unter Hitler. Autoren, Besteller, Leser im Dritten Reich. Berlin: Verlag Galiani 2010, S. 87.

5 Adam: Lesen unter Hitler (Anm. 4), S. 106.

6 Dazu Jan-Pieter Barbian: Literaturpolitik im „Dritten Reich“. Institutionen, Kompetenzen, Betätigungsfelder [1993]. Überarbeitete und aktualisierte Ausgabe. München: Deutscher Taschenbuch-Verlag 1995; Barbian: Literaturpolitik im NS-Staat (Anm. 1).

7 Dienstanweisung für die Lektoren der „Reichsstelle zur Förderung des deutschen Schrifttums“ vom 9. Dezember 1935. Bundesarchiv (BArch) NS 15/144, S. 6. Da exekutive Vollmachten fehlen, hat das Amt Schrifttumspflege seine Verbotswünsche bei staatlichen Instanzen anzumelden. Dass es mit seinen Negativwertungen auf Indizierungsorgane wirkt und die betroffenen Akteure verunsichert, zeigt Dietmar Dürr: Das Amt Rosenberg in der nationalsozialistischen Literaturpolitik. Magisterarbeit Bonn 1994. Positiv begutachtete Werke wurden mit dem Vermerk versehen: „Diese Schrift wird den Gliederungen der N.S.D.A.P., dem Werke ,Kraft durch Freude‘ und allen gleich- 
sowie im seit November 1935 monatlich veröffentlichten Gutachtenanzeiger erscheinen und deren Zahl beachtlich wächst: 1933 sind 25 Mitarbeiter tätigt, 1938 sollen ca. 900, 1940 sogar 14.000 Mitarbeiter beschäftigt sein. ${ }^{8} \mathrm{Zu}$ den Verfassern von Gutachten - deren Zahl bis Ende 1943 auf 60.000 angewachsen sein soll gehört neben später prominenten Literaturwissenschaftlern wie Benno von Wiese auch der schon erwähnte Universitätsgermanist Franz Koch, der von 1936 bis 1943 als ehrenamtlicher ,Hauptlektor` für das neu eingerichtete Fachgebiet Neuere Literatur- und Geistesgeschichte in Rosenbergs Dienststelle arbeitet und dessen Schüler diverse Dissertationen zur Gegenwartsliteratur anfertigen; ${ }^{9}$ mit Ingeborg Drewitz' Doktorarbeit zum völkisch-nationalsozialistischen Autor Erwin Guido Kolbenheyer wird er die letzte Berliner Promotionsschrift in der Zeit des ,Dritten Reiches ${ }^{\text {b betreuen. }} .^{10}$ Der Berliner Fachhistoriker Wolfgang Höppner hat auf Kochs nachhaltige Bemühungen um die literaturwissenschaftliche Erfassung von Gegenwartsliteratur hingewiesen: Im Jahr seiner Berufung nach Berlin veröffentlicht

geschalteten Verbänden zur Anschaffung und Förderung empfohlen.“ Zitiert nach Ernst Piper: Alfred Rosenberg. Hitlers Chefideologe. München: Karl Blessing 2005, S. 339.

8 Die statistischen Angaben finden sich in der Darstellung von Bernhard Payr: Aufgaben des Amtes Schrifttumspflege. In: Hellmuth Langenbucher (Hg.): Die Welt des Buches. Eine Kunde vom Buch. Ebenhausen bei München: Langewiesche-Brandt ${ }^{31942, ~ S . ~ 202-208 ; ~ g e k u ̈ r z t ~ w i e d e r ~ i n ~}$ Joseph Wulf: Kultur im Dritten Reich. Bd. 2: Literatur und Dichtung im Dritten Reich. Eine Dokumentation. Frankfurt/M./Berlin: Ullstein 1989, S. 234-236, hier S. 235 und Fußnote 1.

9 In der Schriftenreihe Stadion. Arbeiten aus dem Germanischen Seminar der Universität Berlin, herausgegeben von Franz Koch, erschienen unter anderem Hans Hermann Schulz: Das Volkstumserlebnis des Arbeitens in der Dichtung von Gerrit Engelke, Heinrich Lersch und Karl Bröger. Würzburg: Triltsch 1940; Hilde Poepping: Der Typus des Deutschen im Werke Karl Kluges. Würzburg: Triltsch 1942. - Im Vorwort zum ersten Band der Reihe hatte der Herausgeber sein Wissenschaftsprogramm dargelegt: „Es wird das Ziel der im Stadion auftretenden Kämpfer sein, die sicheren Gewinne germanistischer Wissenschaft mit jenen methodischen und sachlichen Forderungen in Einklang zu bringen, die das neue Deutschland an die Wissenschaft stellt, um so eine Richtung begründen zu helfen, die aus der Überzeugung von der organischen Einheit und Ganzheit der deutschen Lebenswirklichkeit hervorgeht und Geistiges biologisch zu fassen sucht.“ Franz Koch: Vorwort des Herausgebers. In: Hertha von Ferber: Das Volkstumserlebnis des Joseph Görres. Würzburg: Triltsch 1938, o. S.

10 Ingeborg Neubert, die nach ihrer Heirat Ingeborg Drewitz hieß und zu einer namhaften Schriftstellerin der BRD aufstieg, verfasste ihre Dissertation unter dem Titel Dichterische Gestaltung der ethischen Probleme im Werke E. G. Kolbenheyers; die Disputation fand am 20. April 1945 statt. Zum Promotionsverfahren und der späteren zwiespältigen Verarbeitung in Drewitz' autobiographischem Roman Gestern war Heute vgl. Wolfgang Höppner: Der Berliner Germanist Franz Koch als „Literaturmittler“, Hochschullehrer und Erzieher. In: Gesine Bey (Hg.): Berliner Universität und deutsche Literaturgeschichte. Studien im Dreiländereck von Wissenschaft, Literatur und Publizistik. Frankfurt/M. [u.a.]: Peter Lang 1998 (= Berliner Beiträge zur Wissenschaftgeschichte 1), S. 105-128, hier S. 125-127. 
er die Monographie Gegenwartsdichtung in Österreich (und kann sich danach auch über eine Gratulation des Dichters Josef Weinheber zur Lehrstuhlübernahme in der Reichshauptstadt freuen); ${ }^{11}$ in Zeitschriften berichtet er mehrfach über die aktuelle Textproduktion. ${ }^{12}$ In seinem Einsatz für eine regimekonforme Gegenwartsliteratur verbündet er sich mit dem einflussreichen Rosenberg-Gefolgsmann Hellmuth Langenbucher, dem er am 14. Januar 1937 stolz melden kann: „In meinem Seminar über Roman und Novelle der Gegenwart sitzen Amerikaner u. Engländer in Menge $u$. immer wieder sagt man mir (was uns ja nichts neues ist), daß man von all diesen Namen drüben nichts wisse $u$. höre. Immer wieder kommen Fremde, Ausländer zu mir, um sich gerade über die deutsche Dichtung der Gegenwart zu erkundigen (das Interesse ist sichtlich außerordentlich und allmählich sickert es durch, daß es auch ohne Juden geht) und immer wieder empfehle ich ganz besonders ihr Buch, so daß es sich wohl langsam auch im Ausland durchsetzen wird. Daß es meine Studenten kennen müssen, ist selbstverständlich.“13 Das so formierte „literatur- und wissenschaftspolitische invisible network ${ }^{\text {"14 }}$ zwischen universitärer Literaturforschung und parteiamtlicher Propagierung dient nicht nur dem Informationsaustausch und wechselseitiger publizistischer Unterstützung, sondern auch der Verständigung über literaturhistoriographische Bewertungen. Als Hellmuth Langenbucher 1937 sein erstmals 1933 vorgelegtes Übersichtswerk Volkhafte Dichtung der Zeit überarbeitet, wendet er sich an den professionellen Literaturhistoriker Koch und fragt nach dessen Einschätzung von Schriftstellern. ${ }^{15}$ Die so erworbene Stellung nutzt der Berliner Ordinarius, um Absolventen des Germanischen Seminars auf politischen Be-

11 Franz Koch: Gegenwartsdichtung in Österreich. Berlin: Junker \& Dünnhaupt 1935. Nach seiner Berufung nach Berlin telegraphiert Josef Weinheber am 6. Oktober 1935: „Wieder ein Österreicher in der größeren Welt.“ Hier zitiert nach Höppner: Der Berliner Germanist Franz Koch als „Literaturmittler“, Hochschullehrer und Erzieher (Anm. 10), S. 106.

12 Franz Koch: Umbruch. Ein Forschungsbericht zur Dichtung der Gegenwart. In: Zeitschrift für deutsche Bildung 11 (1935), S. 47-54 und 100-104; F.K.: Zur Dichtung der Gegenwart. Ein Forschungsbericht. In: Zeitschrift für deutsche Bildung 12 (1936), S. 205-210.

13 Franz Koch an Hellmuth Langenbucher. Brief vom 14. Januar 1937. Hier zitiert nach Höppner: Der Berliner Germanist Franz Koch als „Literaturmittler“, Hochschullehrer und Erzieher (Anm. 10), S. 118. Bei dem erwähnten Buch handelt es sich um Langenbuchers Übersichtswerk Volkhafte Dichtung der Zeit (Berlin: Junker \& Dünnhaupt 1933), das 1941 in sechster Auflage erscheint.

14 So prägnant Gerhard Kaiser: Grenzverwirrungen. Literaturwissenschaft im Nationalsozialismus. Berlin: Akademie Verlag 2008, S. 377 [Hervorhebung im Original].

15 Vgl. Höppner: Der Berliner Germanist Franz Koch als „Literaturmittler“, Hochschullehrer und Erzieher (Anm. 10), S. $108 \mathrm{ff}$. 
obachterpositionen unterzubringen: Sein Assistent Paul Stapf, der nach erpresserischen Interventionen seines Doktorvaters Franz Koch gegenüber Julius Petersen als Mitarbeiter der historisch-kritischen Jean-Paul-Ausgabe an die Preußische Akademie der Wissenschaft gekommen ist, arbeitet seit 1937 als Lektor im Amt Schrifttumspflege bei der Reichsleitung der NSDAP. ${ }^{16}$ Elisabeth Frenzel darf nach ihrer Promotion zu Judengestalten auf der deutschen Bühne als Leiterin des Theater-Amts im Amt Rosenberg nach ,jüdisch versippten“ Theaterautoren fahnden; sie gehört zu den letzten Nutzerinnen der Bibliographie des jüdischen Schrifttums, die mit Finanzierung und Unterstützung des RMVP an der Deutschen Bücherei Leipzig erstellt wird und mit dem Observationszeitraum 1901-1940 auch die Gegenwart abdeckt. ${ }^{17}$

An der Deutschen Bücherei in Leipzig arbeitet eine weitere Instanz der NSSchrifttumspolitik: Der von zahlreichen Germanisten und Historikern durchsetzte Sicherheitsdienst der SS versucht ebenfalls das Kultur- und Wissenschaftssystem zu infiltrieren und setzt dazu philologisch geschulte Beobachter der Schrifttumsproduktion ein. $\mathrm{Zu}$ diesen gehören neben dem bereits erwähnten Literaturwissenschaftler Wilhelm Spengler ${ }^{18}$ unter anderen der Wissenschaftsobservator Hans

16 Stapf ist auch an Planungen für das globale Unternehmen ,Mundus‘ beteiligt, die zwischen dem Auswärtigen Amt, dem Reichspropagandaministerium, dem Reichsleiter Presse der NSDAP und dem Reichsfinanzministerium vorgenommen werden, vgl. BArch R 55/1484. Beteiligt sind auch der SD-,Gegnerforscher` Franz Alfred Six, die RMVP-Bürokraten Brauweiler und Haegert; Stapf ist als Geschäftsführer vorgesehen. Nach dem Krieg wird Stapf 1945/46 Leiter der Vorsemesterkurse an der Universität Heidelberg, bis die Militärregierung interveniert. Danach ist er Direktor des Museums Schloss Bernburg. Im September 1952 wechselt er in die Bundesrepublik und geht 1959 in die USA. Zunächst lehrt er als Visiting Professor an der Catholic University in Washington D. C., 1964-74 als ordentlicher Professor an der University of Kentucky, Lexington.

17 Elisabeth Frenzel hatte ihre Dissertation bei Julius Petersen angefertigt und wurde 1938 promoviert. Das Thema hatte ihr nach eigener Aussage der Reichsdramaturg Rainer Schlösser nahegelegt, der nicht nur als Beamter des Goebbels-Ministeriums, sondern auch als Dichter in Erscheinung trat. $\mathrm{Zu}$ diesem gleichfalls promovierten Germanisten, der als Sohn des Weimarer GSA-Archivdirektors Rudolf Schlösser bildungsbürgerlich geprägt war, den Ersten Weltkrieg erlebte und nach 1918 zur Mannschaft um Adolf Bartels stieß, vgl. Ralf Klausnitzer: „Wir rücken die Burgen unseres Glaubens auf die Höhen des Kaukasus“. Reichsdramaturg Rainer Schlösser zwischen Jena-Weimar und Führerbunker. In: Zeitschrift für Germanistik 9/2 (1999), S. 294-317.

18 Wilhelm Spengler ( $\left.{ }^{\star} 1907\right)$, der nach dem Studium der Germanistik, Geschichte, Kunstgeschichte und Philosophie in München und Leipzig 1931 mit der Arbeit Das Drama Schillers bei H. A. Korff promoviert hatte und seit 1934 eine SD-Schrifttumsstelle an der Deutschen Bücherei aufbaute, brachte es nach der Leitung der Abteilung II/21 („Presse und Schrifttum“) bis zum SSStandartenführer und Leiter der Abteilung III C (,Deutsche Lebensgebiete und Kultur“). 
Rössner, ${ }^{19}$ der Presse-Beobachter Walter von Kielpinski ${ }^{20}$ und der ,Volksgeschichtler' Rudolf Levin. ${ }^{21}$ Leiter der SD-Verbindungsstelle an der Deutschen Bücherei in Leipzig wird Martin Nitzsche, der auf der Basis des umfänglichen Materials dieser zentralen Sammelstelle eine Dissertation zum „Deutschlandbild der Emigration“

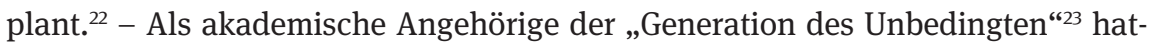
ten diese Geisteswissenschaftler ihre intellektuelle Sozialisation in den krisengeschüttelten 1920er Jahren erfahren. Nach 1933 besetzen sie Führungspositionen in den Weltanschauungs- und Überwachungsinstanzen des NS-Staates - und sind hier für Buchverbote verantwortlich, unter denen die vormaligen Repräsentanten der deutschen Gegenwartsliteratur in besonderem Maße zu leiden haben.

19 Hans Rössner ( $\left.{ }^{\star} 1910\right)$ hatte Germanistik, Geschichte und evangelische Theologie in Leipzig, Graz und Marburg studiert und wirkte bereits zwischen 1934 bis 1936 als hauptamtlicher Referent im SD, war von 1936 bis 1938 Assistent am Germanistischen Seminar der Universität Bonn und promovierte hier 1937 mit der Dissertation Georgekreis und Literaturwissenschaft bei Karl Justus Obenauer. Seit 1938 wieder im SD, wurde er Mitarbeiter in Wilhelm Spenglers Abteilung II/21. Er gilt als Verfasser der Denkschrift Lage und Aufgaben der Germanistik und der deutschen Literaturwissenschaft, die 1938/39 entstand und eine detaillierte Darstellung der institutionellen und konzeptionellen Entwicklung des Faches nach der Machtergreifung gibt. Ab 1940 leitet er die Abteilung III C/3 „Kunst und Volkskultur“ im RSHA. Nach Kriegsende wurde er Lektor beim Stalling-Verlag Oldenburg, Lektor im Insel Verlag und schließlich Leiter des Piper-Verlags in München; er ist an der deutschen Ausgabe von Hannah Arendts Eichmann in Jerusalem beteiligt.

20 Walter von Kielpinski (*1909) studierte von 1929 bis 1934 in Halle, Berlin und Leipzig Germanistik und neuere Sprachen. Nach ehrenamtlicher Tätigkeit an Spenglers Schrifttumsstelle in Leipzig übernahm er ab 1934 im SD die Abteilung II 22 („Presse und Schrifttum“). Seit März 1941 war er Spenglers Stellvertreter in der Kulturabteilung des SD (III C) und betreute die Unterabteilung „Presse, Schrifttum, Rundfunk“.

21 Rudolf Levin ( ${ }^{\star} 1909$ ) studierte Philosophie, Geschichte und Germanistik in Heidelberg, Kiel, München und Leipzig, promovierte 1935 mit der Dissertation Der Geschichtsbegriff des Positivismus unter besonderer Berücksichtigung der rechtsphilosophischen Anschauungen John Stuart Mills und John Austins in Leipzig und beobachtete seit August 1935 im SD die hochschulpolitische Lage, bevor er im RSHA Leiter des Referats VII C 3 („Hexenforschung“) wurde. 1942 versuchte er sich in München mit der Schrift Das magische Problem bei den Gerichtsprozessen des 16. und 17. Jahrhunderts und seine Auswirkungen auf das Volksleben zu habilitieren; die aus seinen Forschungen über ,Hexenprozesse in volksgeschichtlicher Sicht‘ im Rahmen des ,H-Sonderauftrags‘ hervorgegangene Arbeit wurde nach Widerspruch des Zweitgutachters Otto Höfler im Juli 1944 zurückgewiesen. Levin fiel im Februar 1945.

$22 \mathrm{Zu}$ ihm und seiner Dienststelle umfassend Werner Schroeder: ,...eine Fundgrube der Schrifttumsinformation. " Die Arbeitsstelle für Schrifttumsbearbeitung beim Sicherheitshauptamt (SD) und die „SD-Verbindungsstelle an der Deutschen Bücherei“. In: Monika Gibas (Hg): „Arisierung“ in Leipzig. Annäherung an ein lange verdrängtes Kapitel der Stadtgeschichte der Jahre 1933 bis 1945. Leipzig: Leipziger Universitäts-Verlag 2007, S. 116-151, hier S. 127 ff.

23 Michael Wildt: Generation des Unbedingten. Das Führungskorps des Reichssicherheitshauptamtes. Hamburg: Hamburger Edition 2002. 
Wie eine aktuelle und auf umfassenden Archivstudien basierende Arbeit zeigt, stammt der überwiegende Teil von Einzeltitel- und Gesamtverboten, die auf der seit 1935 geführten Liste des schädlichen und unerwünschten Schrifttums verzeichnet werden, vom Reichsführer SS und Chef der deutschen Polizei, der aufgrund der Verordnung des Reichspräsidenten zum Schutz von Volk und Staat auch Buchverbote aussprechen kann: Auf Basis von SD-Informationen (die nicht nur, aber doch vor allem in der Verbindungsstelle zur Deutschen Bücherei gesammelt werden) werden von Himmler in der zweiten Liste des schädlichen und unerwünschten Schriftums mit Stand vom 31. Dezember 1938 insgesamt ca. 1025

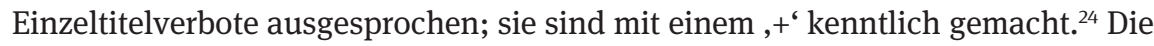
von 1939 bis 1943 in einer Auflage von 7.000 Exemplaren gedruckten Jahreslisten des schädlichen und unerwünschten Schrifttums dokumentieren den wachsenden Einfluss der SD-Beobachter: Von den 126 Einzeltitelverboten des Jahres 1939 werden 90 vom Reichsführer SS verhängt; von den 277 Einzeltitelverboten des Jahres 1940 kommen 203 von ihm; von 182 Einzeltitelverboten des Jahres 1941 kommen 119 von Himmler; und auch die Jahreslisten von 1942 und 1943 dokumentieren, dass Buchverbotsinitiativen mehr oder weniger eindeutig auf die SS und das neugebildete Reichssicherheitshauptamt (RSHA) zurückgehen. ${ }^{25} \mathrm{Ob}$ die auffallende Präsenz der akademisch ausgebildeten SS-,Gegnerforscher` bei der Indizierung von Literatur auf besondere Stellenausstattung oder schärfere Raster zurückgeht, wird noch zu klären sein. Fest steht, dass die jungen SD- und RSHAAkademiker es sogar schaffen, ihre universitären Lehrer in die ,Gegnerforschung zu integrieren. Hier nur knapp zu erwähnen ist der prominente Literaturforscher André Jolles, der mit seinen weitgespannten wissenschaftlichen Interessen der Leipziger Schule der Soziologie um Hans Freyer und Gunther Ipsen nahesteht und durch seinen Studenten Spengler zum SD kommt: Nachdem ihn Wilhelm Spengler im Dezember 1936 mit einem umfangreichen Gutachten empfohlen hatte, ${ }^{26}$ tritt

24 Wieńczysław Niemirowski: Für Führer, Volk und Reich. Schriftsteller und Literaturpolitik im nationalsozialistischen Deutschland. Lublin: Wydawnictwo Uniwersytetu Marii CurieSkłodowskiej 2015, S. 222. Insgesamt enthält die Liste von 1939, die die 1935 von der RSK (ziemlich liederlich) angefertigte Liste ersetzte, 4243 Einzeltitelverbote und 556 Gesamtverbote. Neue Indizierungen von Einzelwerken betrafen u. a. Robert Musils Roman Der Mann ohne Eigenschaften; Verbote des Gesamtwerks galten nun u. a. für Irmgard Keun, Else Lasker-Schüler, Thomas Mann und Carl Zuckmayer.

25 Die 126 Einzeltitelverbote des Jahres 1942 enthalten 98 vom Reichsführer der SS verhängte Indizierungen; 1943 werden 117 Einzeltitelverbote ausgesprochen, von denen 96 von Himmlers Getreuen kommen. Vgl. Niemirowski: Für Führer, Volk und Reich (Anm. 24), S. 222 f.

26 Abgedruckt in Gerd Simon (Hg.): Germanistik in den Planspielen des Sicherheitsdienstes der SS. Ein Dokument aus der Frühgeschichte der SD-Forschung. Teil 1: Einleitung und Text. Tübingen: Verlag der Gesellschaft für Interdisziplinäre Forschung 1998, S. XLI. 
der ausgewiesene Spezialist für „einfache Formen“27 1937 dem Sicherheitsdienst der SS bei. Im März 1941 will Spengler ihn zunächst im Hochschulreferat bei der Zivilverwaltung in Holland verwenden, vermittelt ihm aber dann einen Sonderauftrag für eine Tätigkeit im RSHA: „Obergruppenführer Heydrich hat die beschleunigte Fertigstellung grösserer Denkschriften zu einem bestimmten Fragenkreis der Freimaurerei verfügt, wozu Professor Jolles als Spezialist dringend benötigt wird“, heißt es in einem Schreiben Spenglers an das Reichsministerium für Wissenschaft, Erziehung und Volksbildung (REM), das die Freistellung von seinen universitären Verpflichtungen möglich macht. ${ }^{28}$ Nach ausführlichen Instruktionen durch Franz Alfred Six, Leiter der SD-Zentralabteilungen II/1 (,Weltanschauliche Gegner“) und II/2 („Lebensgebietsmäßige Auswertung“) und den Archivar Paul Dittel erarbeitet Jolles eine umfangreiche Kultur- und Symbolgeschichte der Freimaurerei, deren erster Teil 1944 fertiggestellt ist und als Band 5 der Reihe Quellen und Darstellungen zur Freimaurerfrage im Berliner NordlandVerlag erscheinen soll. Doch bei einem Bombenangriff verbrennt der Satz; im Archiv ungedruckter wissenschaftlicher Schriften der Deutschen Bibliothek in Frankfurt am Main ist nur noch ein Teil des Bürstenabzugs zu finden.

Wie gesagt: Eine umfassende Rekonstruktion der philologisch versierten Literatur-Beobachter in den schrifttumspolitischen Institutionen des NS-Staates ist nicht zu leisten; zumal auch das REM unter Bernhard Rust mitmischt und ebenfalls Gegenwartsautoren begutachtet. ${ }^{29}$ Diesen auf dem Führer-Prinzip beru-

27 André Jolles: Einfache Formen. Legende, Sage, Mythe, Rätsel, Spruch, Kasus, Memorabile, Märchen, Witz. Halle/S.: Max Niemeyer 1930. - Die noch 1999 in unveränderter Form aufgelegte Darstellung wurde in mehrere europäische Sprachen übersetzt und stellt einen proto- oder vielmehr pseudostrukturalistischen Beitrag zur Literaturforschung dar: In Opposition zur Konstruktion geistes- und ideengeschichtlicher Formationen avanciert die „Gestalt“ fundamentaler sprachlicher Äußerungen und die in ihnen sedimentierte „Geistesbeschäftigung“ zu einem Forschungsbereich, zu dessen Beobachtung sich morphologische Prinzipien (die zeitgleich auch in den Naturwissenschaften wieder entdeckt werden) in besonderer Weise eignen. Zeitlich versetzt gleicht sein Entwicklungsgang der politischen Bewegung von Emrich: Einst Mitglied der Sociaaldemocratische Arbeiderspartij (SDAP) und feinfühliger Freund Aby Warburgs (dem er bereits im März 1902 eine genaue Diagnose seiner später ausbrechenden psychischen Erkrankung stellte), tritt er zum 1. Mai 1933 in die NSDAP ein. Aufgrund seiner Parteinahme für die NS-Bewegung verliert Jolles die über alles geliebte Tochter, die sich von ihm abwendet und gemeinsam mit ihrem jüdischen Mann nach Dänemark bzw. Schweden emigriert; zugleich entwickelt er zusehends antisemitische und rassistische Überzeugungen.

28 Wilhelm Spengler an Rudolf Mentzel. Brief vom 9. Dezember 1941. In: Walter Thys (Hg.): André Jolles (1874-1946). „Gebildeter Vagant“. Brieven en Documenten. Amsterdam/Leipzig: Amsterdam University Press/Leipziger Universitätsverlag 2000, S. 890.

29 Als etwa der 1904 in Merseburg geborene Dichter Walter Bauer (der mit der Sammlung Stimme aus dem Leunawerk von 1930 bekannt geworden war) 1936 aus der RSK ausgeschlossen wird, 
henden Organisationen - deren Verhältnisse von der zeithistorischen Forschung als „Polykratie“ und „Ämterchaos“ beschrieben wurde, ${ }^{30}$ während neuere Forschungen von einer „kooperativen Konkurrenz“ ${ }^{31}$ ausgehen - stehen traditionelle Institutionen gegenüber, die sich auf ihre Weise mit der nach wie vor produzierten Gegenwartsliteratur beschäftigen. Neben weiterhin existierenden Zeitungen und Zeitschriften, die trotz unübersehbarer Deformationen eine (fragmentierte) kulturelle Öffentlichkeit herstellen und Neuerscheinungen rezensieren, arbeiten Hochschulen und Universitäten, die nach einer größtenteils von Studenten gestalteten ,revolutionären' Phase an Formen von Selbstverwaltung festzuhalten suchen. Weder die hochfliegenden Pläne zu einer Hochschulreform noch die Idee einer ,politischen Universität‘ im nationalsozialistischen Sinne können verwirklicht werden. ${ }^{32}$ Nicht $\mathrm{zu}$ unterschätzen bleiben schließlich nach wie vor bestehende Kommunikationsgemeinschaften im literarischen Feld wie in

liefert das Ministerium ein Gutachten für den Volksschullehrer, der daraufhin weiter und ohne RSK-Zugehörigkeit veröffentlichen kann (bevor er 1941 wieder ordentliches Mitglied wird). Dazu Niemirowski: Für Führer, Volk und Reich (Anm. 24), S. 215.

30 Zum „Ämterchaos in der Wissenschaftspolitik“ prägnant Michael Grüttner: Wissenschaft. In: Wolfgang Benz/Hermann Graml/Hermann Weiß (Hg.): Enzyklopädie des Nationalsozialismus. Stuttgart: Klett-Cotta 1997, S. 135-153.

31 Zur „kooperativen Konkurrenz“ siehe Stefan Krings: Das Propagandaministerium. Joseph Goebbels und seine Spezialisten. In: Lutz Hachmeister/Michael Kloft (Hg.): Das Goebbels-Experiment. Propaganda und Politik. München: Deutsche Verlags-Anstalt 2005, S. 29-49.

32 Zum „eingespielten Beharrungsvermögen des Lehr- und Forschungsbetriebs“ vgl. Hartmut Tietze: Hochschulen. In: Dieter Langewiesche/Heinz-Elmar Tenorth (Hg.): Handbuch der deutschen Bildungsgeschichte. Bd. V: 1918-1945. Die Weimarer Republik und die nationalsozialistische Diktatur. München: Beck 1989, S. 209-240, Zitat hier S. 229. Versuche von Ministerialbeamten und Professoren, den Einfluß der NSDAP zu begrenzen, um ihre Angelegenheiten selbst zu regeln, beleuchtet Reece C. Kelly: Die gescheiterte nationalsozialistische Personalpolitik und die mißlungene Entwicklung der nationalsozialistischen Hochschulen. In: Manfred Heinemann (Hg.): Erziehung und Schulung im Dritten Reich. Teil 2: Hochschule, Erwachsenenbildung. Stuttgart: Klett-Cotta 1980, S. 61-76. Dass sich ,Reformkonzepte‘ für eine radikale Politisierung der Hochschulen nicht durchsetzen konnten, wurde schon 1936 in einer Bilanz von Walter Groß, Leiter des Rassepolitischen Amtes der NSDAP und dem späteren Wissenschaftsverantwortlichen im Amt Rosenberg deutlich: Die „politische Hochschule“ könne „erst in etwa einem Jahrzehnt verwirklicht werden durch Nachrücken eines weltanschaulich einwandfreien Nachwuchses“, in der Zwischenzeit müsse man ,auf die ,peinlichen Bemühungen“ der derzeitigen Lehrstuhlinhaber, ,Nationalsozialismus zu spielen', verzichten“. Zitiert nach Ahron F. Kleinberger: Gab es eine nationalsozialistische Hochschulpolitik? In: Heinemann (Hg.): Erziehung und Schulung im Dritten Reich. Teil 2 (Anm. 32), S. 9-30, hier S. 11. Dazu auch: Volker Losemann: Reformprojekte nationalsozialistischer Hochschulpolitik. In: Karl Strobel (Hg.): Die deutsche Universität im 20. Jahrhundert. Die Entwicklung einer Institution zwischen Tradition, Autonomie, historischen und sozialen Randbedingungen. Vierow: SH-Verlag 1994, S. 97-115. 
den weiterhin privatwirtschaftlich organisierten Verlagen. Auch wenn diese von NS-Aktivisten und NSDAP-Mitgliedern partiell eingenommen werden, bleibt im Einzelfall zu prüfen, wie sich ästhetische Geltungsansprüche und Reputationshierarchien unter deren Einfluss relativieren oder von ihnen dominiert werden können. Ebenfalls zu rekonstruieren ist die „kooperative Konkurrenz“ zwischen den Institutionen zur Beobachtung und Kontrolle von Gegenwartsliteratur. So agiert die Schrifttumsabteilung des RMVP im Bestreben der um Joseph Goebbels zentrierten Kultus-Bürokratie, im Konkurrenzkampf mit anderen Instanzen der NS-Schrifttumspolitik ,mithalten“ und Positionen sichern zu können: Nachdem der Sicherheitsdienst der SS eine eigene Verbindungsstelle an der Deutschen Bücherei in Leipzig betreibt und mit Wilhelm Spengler einen promovierten Literaturwissenschaftler beschäftigt, der später zum ranghöchsten Germanisten unter den im SD beschäftigten ,Gegnerforschern“ avanciert, will auch die Schrifttumsabteilung an dieser zentralen Sammelstelle beobachten - und richtet deshalb das von Wilhelm Emrich ab dem 1. Januar 1942 besetzte ,Erkundungsreferat an der Deutschen Bücherei ein. Den hier praktizierten Beobachtungen ist nun nachzugehen.

\section{Gegenwartsliteratur im Prüfverfahren: Die Schrifttumsabteilung des Reichspropagandaministeriums}

Zahlreiche promovierte und habilitierte Geisteswissenschaftler arbeiten im Reichspropagandaministerium unter dem promovierten Literaturwissenschaftler Joseph Goebbels. Dessen Geschichte ist noch nicht geschrieben und kann auch hier nicht skizziert werden. ${ }^{33}$ Knappe Hinweise auf Organisation, Personal und Arbeitsweisen in der Abteilung Schrifttum (VIII) im RMVP sollen den Rahmen abstecken, um die Observationen von Gegenwartsliteratur im Spannungsfeld politischer Lenkungsansprüche rekonstruieren zu können.

Die im Oktober 1934 in das RMVP eingegliederte Abteilung VIII (Schrifttum) soll das gesamte deutschsprachige Schrifttum aus dem In- und Ausland über-

33 Die ursprünglich sieben Abteilungen des RMPV wurden bis zum Jahr 1941 auf siebzehn erweitert; in ihnen arbeiteten im Mai 1942 rund 1500 Beamte, Angestellte und Arbeiter. Bildungsund mentalitätsgeschichtliche Hintergründe des Ministers und seiner Mitarbeiter rekonstruieren die Beiträge in Hachmeister/Kloft (Hg.): Das Goebbels-Experiment (Anm. 31). 
wachen. ${ }^{34}$ Dazu beobachtet die Abteilung nicht nur das Verlagswesen, sondern auch den Buchhandel und die Büchereien (Staats-, Landes, Universitäts- und Stadtbibliotheken sowie den Leihbuchhandel). Gegliedert ist sie in drei Hauptreferate („Deutsches Schrifttum: Inland“, „Deutsches Schrifttum: Ausland“, „Büchereiwesen“), die zunächst die Bereiche der Buchproduktion und des Buchexports, der Lesungen und Buchausstellungen im In- und Ausland sowie die Verbreitung propagandistischen und schöngeistigen Schrifttums prüfen und durch Festlegung von buchpolitischen Richtlinien reglementieren. ${ }^{35}$ Überwacht werden ebenfalls die Leihbüchereien sowie Preisverteilungen und buchbezogene Stiftungen. Ab 1938 ist die Abteilung VIII für Verbotsauflagen verantwortlich und übernimmt damit Aufgaben, die vorher die RSK innehatte. Zensurvollmachten waren freilich schon vorher übertragen worden: Seit 1936 erteilt die Schrifttumsabteilung die Genehmigungen für Übersetzungen, ab 1937 gibt es die Anmeldepflicht von Schrifttumspreisen. Kontroll- und Indizierungsfunktionen realisiert die Schrifttumsabteilung durch explizite Verbote, die in den seit 1939 periodisch aktualisierten Listen des schädlichen und unerwünschten Schrifttums abgedruckt werden sowie in Kooperation mit der Wirtschaftsstelle des Deutschen Buchhandels (die seit 1939 eine Art Vorzensur ausübt). „Mit der Eingliederung dieser bedeutenden Lenkungsaufgaben erreichte die Schrifttumsabteilung die Position einer autoritativen Überwachungs- und Zensurbehörde des Dritten Reiches.“36

Die quantitativen Aspekte dieser Aufgaben sind nicht zu unterschätzen: Folgt man der älteren Darstellung von Dietrich Strothmann, werden 1940 von der Schrifttumsabteilung im RMVP ca. 2.500 Verleger, 23.000 Buchhandlungen, 3.000 Schriftsteller, 20.000 Neuerscheinungen im Jahr sowie über eine Million im Handel erhältliche Buchtitel und über fünfzig reichsweite Literaturpreise beaufsichtigt; zugleich kontrolliert man über tausend Kundgebungen zu Schrifttumsfragen und Tausende von Autorenlesungen. ${ }^{37}$ Seit dem 30. Juni 1933 untersteht auch die Deutsche Bücherei Leipzig dem RMVP. Neben der Deutschen Bücherei als der zentralen Sammelstelle der deutschsprachigen Buchproduktion gehört auch die Reichsschrifttumsstelle zum Geschäftsbereich; sie war 1934 für die Buchpropaganda geschaffen worden und wird später in „Werbe- und Beratungsamt für das deutsche Schrifttum“ umbenannt.

34 Bei diesem Vorgang wurde das Referat für Literatur- und Verlagswesen aus der Abteilung „Propaganda“ ausgegliedert und zur achten Abteilung des Propagandaministeriums erhoben; vgl. Barbian: Literaturpolitik im „Dritten Reich“(Anm. 6), S. 172.

35 Dietrich Strothmann: Nationalsozialistische Literaturpolitik. Ein Beitrag zur Publizistik im Dritten Reich. Bonn: H. Bouvier u.Co.Verlag 1960, S. 23.

36 Strothmann: Nationalsozialistische Literaturpolitik (Anm. 35), S. 24

37 Strothmann: Nationalsozialistische Literaturpolitik (Anm. 35), S. 27. 
Wie in verschiedenen anderen Bereichen besteht auch in der Abteilung VIII eine Art Personalvernetzung mit anderen staatlichen und parteilichen Ämtern zur Überwachung des Buch- und Schrifttumswesens. So ist der jeweilige Leiter der Schrifttumsabteilung im RMVP zugleich Vorsitzender der Reichsschrifttumsstelle und stellvertretender Präsident der RSK. ${ }^{38}$ Heinrich Wismann, der die Abteilung von 1934 bis 1937 leitet und wegen einer halbjüdischen Ehefrau (mit der er bis 1934 verheiratet gewesen war) entlassen wird, gehört zugleich dem Auktionsausschuss des Börsenvereins des deutschen Buchhandels und dem Vorstand des Reichsverbandes deutscher Schriftsteller an.

Die Schrifttumsabteilung leidet unter ständigem Personalmangel und Leitungswechseln. Auffällig ist die hohe Zahl promovierter Geisteswissenschaftler. So arbeiten im Hauptreferat I unter Johannes Schlecht in vier Einzelreferaten unter anderen Dr. Hugo Koch (im Referat 2 „Buchverbotswesen“), Dr. Hans Wilhelm Hagen sowie Dr. Wilhelm Emrich (der sich 1944 habilitiert). Im Hauptreferat II arbeiten unter Dr. Rudolf Erckmann vier Einzelreferate zur „Überwachung und Förderung des gesamten Verlagswesens“. Hauptreferat III unter Leitung von Paul Hövel beschäftigt unter anderem Dr. Erich Kühne (der nach 1945 Karriere in der DDR macht und Professor in Rostock wird) sowie Dr. Wilhelm Ruoff, der zuvor Lektor der Deutschen Akademie war und später als Schriftleiter der Zeitschrift Europäische Literatur reüssieren wird.

Für die Tätigkeit dieser geisteswissenschaftlichen Literatur-Beobachter relevant sind die Weichenstellungen, die nach Übernahme der Leitung durch Wilhelm Haegert vorgenommen werden: Zwischen November 1939 und Oktober 1944 konsolidiert sich die Abteilung VIII; ihr wird die gesamte Buchzensur zugewiesen; die Kooperation mit dem Reichswirtschaftsministerium in der Frage der Papierzuteilung sichert weiteren Einfluss. ${ }^{39}$ Im Frühjahr 1943 erfolgen Planungen zur Einrichtung einer „zentralen Schrifttumsagentur“, wogegen die Haushaltsabteilung des RMVP keine Bedenken hat und mit der auch der Minister J. Goebbels einverstanden ist; Referent Heinrich Gruber wird für deren Leitung freigegeben und am 29. Dezember 1943 zum Chef der vom RMVP alimentierten Deutschen

38 Die Leiter der Abteilung VIII (Schrifttum) waren in chronologischer Folge ihrer Amtsführung: 1934-37 Heinrich/Heinz Wismann, 1937-38 Karl Heinz Hederich, 1938-1939 Alfred-Ingemar Berndt, 1939-1944 Wilhelm Haegert, 1944 bis Kriegsende Wilfried Bade.

39 Im Oktober 1939 wird die Wirtschaftsstelle des deutschen Buchhandels zum Zensurinstrument durch die Bewirtschaftung der Papier- und Einbandsstoffe für die Buchverlage. Die inhaltliche Prüfung erfolgt durch die Schrifttumsabteilung des RMVP; siehe dazu Barbian: Literaturpolitik im NS-Staat (Anm. 1), S. $275 \mathrm{f}$. 
Buchagentur befördert. ${ }^{40}$ Diese Agentur, die „besonders kriegswichtige Aufgaben“ zur „Verbreitung des deutschen Schrifttums im Auslande“ erfüllen soll, ${ }^{41}$ residiert in der Wirtschaftsstelle des deutschen Buchhandels in der Berliner Friedrichstraße 31 - ein weiterer Hinweis auf die engen Verflechtungen zwischen Ministerialbürokratie und Buchwirtschaft. Ihre wichtigste Aufgabe besteht in der Vermittlung von Übersetzungsrechten zwischen den deutschen Verlegern und denen der verbündeten und neutralen Länder. ${ }^{42}$

Die in Nazi-Deutschland produzierte Gegenwartsliteratur und ihre wissenschaftliche Reflexion bleiben in nicht zu unterschätzender Weise von den Kontroll- und Indizierungsfunktionen der Schrifttumsabteilung abhängig. Auch diese Verhältnisse sind spannungsreich und konfliktträchtig: Einerseits können die Angestellten des Reichspropagandaministeriums die Maßnahmen zur Überwachung des im Reich zirkulierenden Schrifttums zumindest teilweise erfolgreich durchsetzen; andererseits suchen Autoren und Verleger sowie Angehörige und Institutionen der scientific community ihre Betätigungsfelder auch unter den Bedingungen der ,Gleichschaltung‘ zu bewahren. Deutlich werden diese Spannungen zwischen politischen Lenkungsansprüchen und einem partiell autonomen Handeln von Autoren und Verlegern vor allem in den verschiedenen Maßnahmen zur Reglementierung der Buchproduktion - die mit der im März 1940 erlassenen und am 1. April 1940 in Kraft tretenden Anweisung zur „Anmeldungen von Verlagsplanungen“ eine neue Stufe erreichen ${ }^{43}$ - sowie in der Tätigkeit von Staats-, Landes- und Hochschulbibliothekaren, die bei aller Sympathie für die ,nationalsozialistische Revolution“ ihre Sammlungsgebiete vor Vernich-

40 Stellungnahme der Haushaltsabteilung zur Einrichtung einer zentralen Schrifttumsagentur vom 28. Juni 1943. BArch R 55/170, Bl. 179; Einverständnis von Minister J. Goebbels. BArch R 55/ 170, Bl. 181.

41 Vermerk RMVP vom 24. April 1944 und vom 20. Mai 1944. BArch R 55/170, Bl. 31 und 32.

42 Schon vorher spielt Gruber eine unrühmliche Rolle bei der Arisierung des Wiener Paul Zsolnay-Verlages, wogegen Rechtsanwälte sich beim RMVP beschweren; die Beschwerde wird jedoch zurückgewiesen.

43 Die im März 1940 erlassene und am 1. April 1940 in Kraft tretende Anweisung zur „Anmeldung von Verlagsplanungen“ kam von Wilhelm Haegert, dem Leiter der Abteilung Schrifttum im Reichsministerium für Volksaufklärung und Propaganda und verpflichtete die Verleger zur detaillierten Offenlegung ihrer Programme: „Unter dem Kennwort „Planungs-Anmeldung‘ sind möglichst genaue Angaben über den Verfasser und den vorläufigen Titel sowie eine knappe Übersicht über Inhalt und voraussichtlichen Aufbau, über Umfang, vorgesehene Auflage und voraussichtlichen Ladenpreis der Werke einzureichen. Erwünscht ist in jedem Fall die Angabe der Gründe, die den Verlag zur Herausgabe gerade dieses Werkes während des Krieges veranlassen." Vertrauliche Mitteilung für die Fachschaft Verlag, Nr 49 vom 27. März 1940, S. 1; hier zitiert nach dem Abdruck in Handbuch der Reichsschrifttumskammer. Hrsg. von Wilhelm Ihde unter Mitarbeit von Günther Gentz. Leipzig 1942: Verlag des Börsenvereins der Deutschen Buchhändler, S. 137-139. 
tung zu bewahren suchen und die nun als „schädlich[] und unerwünscht[]“ geltende Literatur weiterhin sichern wollen. Die (auch von amtlicher Seite unterstützte) Bewahrung der Archivfunktionen und der damit verbundene Schutz von Büchern - etwa vor dem Eingriff von NS-Aktivisten, die mit den Bücherverbrennungen im Frühjahr 1933 ihre Umgangsformen mit der Überlieferung gezeigt hatten - bedeutet nämlich nicht, dass die Behandlung verbotener Literatur und des ,weltanschaulichen Gegners‘ geregelt ist. Nach den ersten Aktionen der Jahre 1933/34 werden seit 1935 koordinierte Maßnahmen zur Absonderung weltanschaulich inkompatibler Literatur ergriffen, die allerdings die beteiligten Akteure aufgrund des Fehlens an exakten Vorgaben vor Herausforderungen stellen. So verpflichtet der Runderlass des REM vom 3. April 1935 die Bibliotheken zur genauen Überprüfung ihrer Bestände und zur ,Sekretierung“ des „schädlichen und unerwünschten Schrifttums“; unklar aber bleibt, was diesem Schrifttum im Einzelnen zuzurechnen und auszusondern ist. Im März 1936 ergeht in einem vertraulichen Rundschreiben des Reichsministeriums für Wissenschaft, Erziehung und Volksbildung an die Unterrichtsverwaltungen der Länder schließlich die Liste 1 des schädlichen und unerwünschten Schrifttums, die den Bibliotheken den Auftrag gibt, ihre Buchbestände genau zu überprüfen und verbotene Literatur unter Verschluss zu halten. Gleichwohl bleiben auch hier die Erwartungen hinter der Realität zurück; selbst Werke marxistischer und anarchistischer Theoretiker und offiziell verfemter ,Asphaltliteraten' stehen zunächst weiterhin frei zur Verfügung. Nachdrückliches Drängen des REM führt zwar zur Sperrung von Titeln für die allgemeine Ausleihe; gleichwohl bleiben etwa in der Deutschen Bücherei in Leipzig indizierte Werke im öffentlich zugänglichen Hauptkatalog (während andere Bibliotheken die Titelaufnahmen aus den Gesamtkatalogen entnehmen und nur noch in einem der Öffentlichkeit unzugänglichen Dienstoder Sonderkatalog erfassen). Verbotene Literatur darf danach nur benutzt werden, wenn die Einsichtnahme mit wissenschaftlicher Arbeit zusammenhängt.

Von noch weiterreichender Bedeutung sind freilich die Kontroll- und Indizierungsfunktionen der Schrifttumsabteilung in Bezug auf die aktuelle Buchproduktion und -distribution. Hervorzuheben bleibt die Deutsche Bücherei (DB) in Leipzig als institutioneller Einsatzort, an dem die Überwachung der eingehenden Pflichtexemplare gleichsam unter den Augen des RMVP abläuft. Die seit Juni 1933 dem Goebbels-Ministerium unterstehende DB hat dabei eine wachsende Zahl von Aufgaben zu erfüllen; ein stetig erhöhter Zugang von Publikationen ist mit immer weniger Mitarbeitern zu bewältigen. Zudem leidet die Arbeit der DB unter ungeklärten Sammelgrundsätzen, die mehrfach vom RMVP eingefordert werden; erst im Laufe des Jahres wird ein sechsseitiges Dokument mit Sammelgrundsätzen fertiggestellt, das rückwirkend zum 1. Januar 1943 in Kraft 
tritt. ${ }^{44}$ Hier besetzt der in Frankfurt am Main promovierte Philologe Wilhelm Emrich seit dem 1. Januar 1942 das ,Erkundungsreferat' und übernimmt damit scheinbar geringe, doch nicht $\mathrm{zu}$ vernachlässigende Funktionen im politisch reglementierten Handeln mit der Gegenwartsliteratur. Wie wichtig seine Tätigkeit ist, zeigt sich schon an dem Umstand, dass seine Einstellung im Dezember 1941 trotz einer für das gesamte Ministerium ,allgemein verfügten Einstellungssperre“ durch den Reichsminister selbst genehmigt wird (der zugleich betont, dies sei „die letzte Genehmigung“, die er vorläufig ausspreche): Nachdem der Staatssekretär im Propagandaministerium Leopold Gutterer darauf gedrängt hatte, die „Erfassung des staatsfeindlichen Schrifttums zu aktivieren“ und auf seine Weisung bereits im Vorfeld das Hauptreferat „Buchverbotswesen“ der Schrifttumsabteilung angehalten worden war, ein „Erkundungsreferat“ in der DB in Leipzig einzurichten, das „die laufende Überwachung aller deutschsprachigen Neuerscheinungen übernehmen“ sollte, avanciert Wilhelm Emrich zum Inhaber dieser Verbindungstelle. ${ }^{45}$

Emrichs Tätigkeiten als ,Erkundungsreferent‘ an der DB Leipzig gehören zu den vielfältigen Bemühungen der NS-Kultusbürokratie um eine effektive Kontrolle der Schrifttumsproduktion. Zugleich stehen sie in nicht einfach zu durchschauenden Verhältnissen mit den Anstrengungen der DB um Sicherung ihrer bibliothekarischen Bedeutsamkeit. Denn die 1912 auf Initiative des Börsenvereins des deutschen Buchhandels gegründete DB - die 1916 ihren noch heute am Deutschen Platz befindlichen Hauptsitz bezog - war eine Buchsammlungsstätte ohne Geschichte: Vor allem im Vergleich mit den anderen großen Bibliotheken des Reiches wie der Preußischen Staatsbibliothek Berlin oder der Bayrischen Staatsbibliothek München hatte die DB keine historisch langfristigen Traditionen und auch keine spezifischen Sammlungen; sie krankte also seit ihrer Gründung an ,Minderwertigkeitskomplexen‘. Diese Defizite suchten die Generaldirektoren (zu denen auch der Kleist-Forscher Georg Minde-Pouet gehörte) durch Übernahme großangelegter bibliographischer Missionen wett zu machen - was dazu führte, dass man an der DB neben der Deutschen Nationalbibliographie auch andere umfassende Bücherverzeichnisse und Kataloge erstellte; nach 1933 unter ande-

44 Vgl. BArch R 55/642. An den Sitzungen zur Regelung des Haushalts der DB ist Emrich nicht beteiligt; an ihr nehmen Generaldirektor Uhlendahl, RMVP-Ministerialdirigent Dr. Ott, RMVPReferent Dr. Hans Gerber, Stadtkämmerer Dr. Lisse und andere teil.

45 Leiter der Abteilung Schrifttum des RMVP an den Leiter der Personalabteilung des RMVP vom 4.12.1941. BArch Berlin, ehemaliges Berlin Document Center (BDC), RK I 95, Bl. 2244; Leiter der Personalabteilung des RMVP an den Minister vom 22.12.1941. BArch Berlin, ehemaliges BDC, Bl. 2258; Büro des Staatssekretärs an den Leiter der Personalabteilung des RMVP vom 24.12.1941. BArch Berlin, ehemaliges BDC, Bl. 2264. 
rem die periodisch aktualisierte Jahresliste des verbotenen und unerwünschten Schrifttums, die Bibliographie der ausländischen Werke über Deutschland sowie die Liste der in der DB unter Verschluss gestellten Druckschriften.

An der DB hatte der SD-Mitarbeiter Wilhelm Spengler schon 1934 eine eigene Beobachtungsstelle eingerichtet. Der promovierte Literaturwissenschaftler, der bis zum SS-Standartenführer aufsteigen sollte, schildert seine Tätigkeit nach 1945 mit betonter Naivität: „Jede Woche kamen Neuerscheinungen herein. Sollte irgend jemand zum Professor ernannt werden, dann hat die Parteikanzlei rückgefragt. Ich las dann das gesamte Schrifttum von demjenigen durch, wie er sich, die ganze Persönlichkeit entwickelt hat. “46 Auch die Parteiamtliche Prüfungskommission zum Schutze des NS-Schrifttums (PPK) unter der Leitung von Philipp Bouhler stützte sich bei ihrer Arbeit zur Bekämpfung des nach der Machtübernahme florierenden und sich nationalsozialistisch gebärdenden ,Konjunkturschrifttums` auf die DB, in der sie eine eigens eingerichtete Außenstelle unterhielt. ${ }^{47}$ In eiligen und vom Reichsminister beförderten Vorgängen installierte die Schrifttumsabteilung des RMVP im Dezember 1941 ein Erkundungsreferat in Leipzig, das Wilhelm Emrich besetzen sollte.

Warum sowohl SD und PPK als auch die Schrifttumsbeobachter des RMVP bei ihrer Indizierungspraxis die DB und nicht deren Schwesteranstalten in Berlin und München nutzten, wird angesichts der Sammlungstätigkeit der DB und deren Position im Bibliothekswesen Deutschlands plausibel. Während die Staats-, Landes-, Universitäts- und Stadtbibliotheken das Auswahlprinzip vertraten, folgte die DB dem Grundsatz der Vollständigkeit. Wie ihr Direktor Heinrich Uhlendahl 1938 betonte, war die nationale Buch- und Zeitschriftenproduktion der letzten

46 Vernehmungsprotokoll des Dr. phil. Wilhelm Spengler vom 16. Mai 1947, hier zitiert nach Schroeder: ,„... eine Fundgrube der Schrifttumsinformation.“(Anm. 22), S. 116.

47 Der PPK gelang es zwar, ihre Kontroll- und Überprüfungsansprüche über die parteinahe Literatur hinaus auf das wissenschaftliche, pädagogische und volksbildnerische Schrifttum auszudehnen; jedoch scheiterte sie 1937/38 mit dem Versuch, ein ihrer Aufsicht unterstehendes zentrales Schrifttumsamt der NSDAP zu schaffen und sich den Börsenverein und die Reichsschrifttumskammer zu unterstellen; vgl. Reinhard Bollmus: Parteiamtliche Prüfungskommission zum Schutze des nationalsozialistischen Schrifttums (PPK). In: Wolfgang Benz/Hermann Graml/ Hermann Weiß (Hg.): Enzyklopädie des Nationalsozialismus. München: Klett-Cotta ${ }^{21998,}$ S. 634f. Zur PPK siehe auch Karsten Jedlitschka: Die „Parteiamtliche Prüfungskommission zum Schutze des nationalsozialistischen Schrifttums“ - Zensurfelder und Arbeitsweise am Beispiel des Münchner Lektors Ulrich Crämer. In: Archiv für Geschichte des Buchwesens 62 (2008), S. 231-226; Barbian: Literaturpolitik im NS-Staat (Anm. 1), S. 162-179. Die seit Frühjahr 1935 bestehende PPKDependance an der DB ermöglichte den PPK-Beamten zwar auch die Kontrolle der Verlagsproduktion unter dem Gesichtspunkt der Zensur, diente aber in erster Linie als „bibliographische Auskunftsstelle“ und Plattform für die Erstellung der „Nationalsozialistischen Bibliographie“; Barbian: Literaturpolitik im NS-Staat (Anm. 1), S. $167 \mathrm{f}$. 
25 Jahre „in der Deutschen Bücherei in einer Vollständigkeit vorhanden, wie sie keine Nationalbibliothek eines anderen Landes für ihr Schrifttum aufweisen“ könne. ${ }^{48}$ Ihr ging als einziger Bibliothek im Reich die deutsche Verlagsproduktion weitgehend kostenlos zu. Das 1935 durch die Reichskulturkammer erlassene Reichspflichtexemplargesetz steigerte den Selbstanspruch der DB als Zentralort zur systematischen Erschließung und Sicherung der nationalen Kultur- und Wissensbestände nachhaltig. In Konkurrenz zur Preußischen Staatsbibliothek hatte sie sich zudem zum bibliographischen Zentrum Deutschlands entwickelt und gewann als reichsweit agierende Auskunftsstelle zunehmend an Bedeutung. 49

Den NS-Buchbeobachtern war die Bedeutung der DB also frühzeitig bewusst. Dass das Reichspropagandaministerium jedoch erst 1941 ein eigenes „Erkundungsreferat“ einrichtete, könnte an einem in diesem Jahr eskalierenden Streit zwischen der PPK und dem Goebbels-Ministerium gelegen haben. Bei dieser Auseinandersetzung ging es um die Richtlinienkompetenz bei der Beschlagnahme und dem Verbot von Druckschriften: Nach einem persönlichen Vortrag des ehrgeizigen Bouhler hatte Hitler im März 1941 noch einmal ausdrücklich bestätigt, dass die PPK ebenso wie das RMVP bereits erschienene Bücher verbieten und bei der Gestapo Anträge auf deren Beschlagnahmung stellen durfte. ${ }^{50}$ Bei Neuerscheinungen oder im Druck befindlichen Werken galten Anträge der Parteiamtlichen Prüfungskommission auf Einziehung als genehmigt, wenn das Propagandaministerium nicht innerhalb von drei Wochen dagegen interveniert hatte. Dass Bouhlers Kommission zudem ein Stimmrecht in dem 1941 vom RMVP eingesetzten Ausschuss zur Vergabe von Papierkontingenten erhielt und auf diese Weise über die Publikationsprojekte sämtlicher Verlage mitentscheiden konnte, steigerte die Konfusion - und mag dazu geführt haben, dass das RMVP mit der Errichtung einer eigenen Dependance an der DB nach einem Weg suchte, sich exklusive Grundlagen für seine Indizierungspraxis zu verschaffen. Um be-

48 Beitrag Uhlendahls (Manuskript): Zum 25. Geburtstag der Deutschen Bücherei, 1.1.1938. Archiv der Deutschen Nationalbibliothek Leipzig (ADNBL) 182/3, Bd. I (1928-1944), Bl. 277.

49 Die wachsende Bedeutung der Auskunftsstelle dokumentieren die jährlichen Verwaltungsberichte der DB. Ihnen ist zu entnehmen, dass nach bescheidenen Anfängen im Jahr 1915 (64 Auskünfte), 1925 bereits 2.000, 1935 jedoch 36.647 Auskünfte erteilt wurden. Bis 1942 setzte sich dieser Aufwärtstrend fort: 1936 (35.666), 1937 (40.242), 1938 (48.106), 1939 (47.846), 1940 (68.855), 1941 (74.215), 1942 (63.842). Vgl. Dritter Bericht über die Verwaltung der Deutschen Bücherei des Börsenvereins der Deutschen Buchhändler zu Leipzig im Jahr 1915. Leipzig 1916 ff.; Manuskripte für die Verwaltungsberichte 1938-1942. ADNBL 181/1-1938 bis 181/1-1942. Die Auskünfte wurden zu einem großen Teil an Privatpersonen erteilt, gleichwohl zählten auch militärische, wissenschaftliche, staatliche und industrielle Institutionen zu den Adressaten.

50 Barbian: Literaturpolitik im NS-Staat (Anm. 1), S. $176 \mathrm{f}$. 
stehende Informationsvorsprünge von PPK und SD auszugleichen und die „Erfassung des staatsfeindlichen Schrifttums“ zu intensivieren, sollte der neue Referent des RMVP „nun bei der Deutschen Bücherei die laufende Überwachung aller deutschsprachigen Neuerscheinungen übernehmen“..51 In diesem Zusammenhang untersagte das Propagandaministerium der DB zunächst auch eine räumliche Erweiterung der SD-Verbindungsstelle in der DB, da man selbst Probleme hatte, dort einen geeigneten Arbeitsplatz für seinen neuen Referenten Emrich zu finden - er bezog schließlich einen Raum in der „Traditionsbibliothek von 1848 “.52

Der so installierte Wilhelm Emrich legt am 19. Januar 1942 vor dem Generaldirektor der DB Heinrich Uhlendahl den Diensteid ab („Ich werde dem Führer des Deutschen Reiches und Volkes Adolf Hitler treu und gehorsam sein und meine Dienstobliegenheiten gewissenhaft und uneigennützig erfüllen.") und übernimmt die Überwachung des in die Bibliothek eingehenden deutschsprachigen Schrifttums (vor allem aus der Schweiz) sowie die Betreuung der hier erstellten Bibliographie des jüdischen Schrifttums in deutscher Sprache (BjS). ${ }^{53}$ Diese ,Judenbibliographie“ ist vor dem Hintergrund der umfassenden Erfassungs- und Katalogisierungsaufgaben der DB zu sehen; sie erwächst andererseits aus dem Bestreben, klare Rubrizierungsmöglichkeiten für den staatlich verordneten Antisemitismus im Umgang mit Literatur zu gewinnen. Nachdem im April 1940 eine Totalindizierung der Schriften aller ,voll- und halbjüdischen“ Autoren verhängt worden war, erhält die DB vom Reichspropagandaministerium im Juni 1941 den Auftrag, ein „Gesamtverzeichnis des jüdischen Schrifttums in deutscher Sprache“ zu erstellen. Das Reichspropagandaministerium stellt Sondermittel in Höhe von 27.200 RM bereit und so beginnen unter der Leitung von Bibliothekar Hans Ruppert am 1. August 1941 die Arbeiten. Bis zum März 1942 werden insgesamt 28.000 Karteikarten angelegt, die für Auskunftserteilungen an staatliche Behörden und Parteidienststellen nutzbar sind. Im Frühjahr 1944 müssen die Arbeiten aufgrund kriegsbedingten Personalmangels eingestellt werden.

51 Leiter der Abteilung Schrifttum des RMVP an den Leiter der Personalabteilung des RMVP vom 4.12.1941. BArch Berlin, ehemaliges BDC, RK I 95, Bl. 2244.

52 Entwurf einer Niederschrift Heinrich Uhlendahls über die 1. Vorbesprechung des Haushaltsplans der DB für das Rechnungsjahr 1942, 11.12.1941, BArch R 55/642, Bl. 64-74, hier Bl. 74; Günther Lutz (RMVP) an Uhlendahl, 28.5.1942. ADNBL 154/5 (Hefter Ley-Ly), Bl. 337.

53 Dienstleistungszeugnis für Wilhelm Emrich vom 12. April 1944. BArch Berlin, ehemaliges BDC: „Zunächst wurde er vom 1. Januar 1942 bis 31. Januar 1943 in der Deutschen Bücherei in Leipzig eingesetzt, wo ihm die Überwachung des gesamten neu eingehenden deutschsprachigen Schrifttums und die Betreuung der Judenbibliographie oblag.“ 
Wie die nur dürftigen Quellen im Hausarchiv der DB in Leipzig belegen, wurden Planungen zur Erstellung dieser Bibliographie des jüdischen Schrifttums in deutscher Sprache seit April 1941 verfolgt und durch den DB-Bibliotheksinspektor Curt Fleischhack an den RMVP-Referatsleiter Hugo Koch für eine Ministervorlage übermittelt. ${ }^{54} \mathrm{Da}$ man den „Anteil der Juden am deutschen Schrifttum (den Begriff als rassische Volljuden genommen)“ auf sechs Prozent schätzte, errechnete man einen Anteil am Buchhandelsschrifttum von rund 77.000 Schriften (von insgesamt 1.290.000 Schriften) für den Zeitraum von 1901 bis $1940 . .^{55}$ Die vom Leiter der Schrifttumsabteilung kommende Ministervorlage vom 24. Juni 1941 nahm diese Vorschläge auf und begründete den Ressourceneinsatz mit dem „Fehlen eines zuverlässigen Verzeichnisses der jüdischen Schriften“ und der Notwendigkeit eines Katalogs, dessen „Vorarbeiten durch wissenschaftlich geschulte Bibliothekskräfte in der Deutschen Bücherei“ durchzuführen seien. Zugleich erhöhte man die Zahlen: „Sachverständige der Deutschen Bücherei schätzen den Anteil der jüdischen Autoren an den im Zeitraum von 1901-1940 erschienenen 2.180.000 Schriften auf etwa 90.000. “56 Das Reichspropagandaministerium bewilligte schließlich jährlich 27.200 RM für die „Erstellung der Bibliographie des jüdischen Schrifttums 1901-1940“. Doch die im August 1941 aufgenommene Arbeit erwies sich aus mehreren Gründen als schwierig: Zum einen gab es unterschiedliche Instanzen der Bevölkerungsüberwachung (unter anderem das Reichssippenamt, der NS-Rechtswahrerbund, das Zentralinstitut für Geschichte des Neuen Deutschland, die Antisemtische Aktion und das Jüdische Zentralmatrikelamt Prag, obwohl dort „viel urkundliches Material bei der Aktion gegen die Synagoge im Herbst 1938 in Verlust geraten ist“..) ${ }^{57}$ Zum anderen erfolgte die Katalogisierung von Autoren auf der Basis von Quellen, deren Fragwürdigkeit den Mitarbeitern selbst bewusst waren: Zurückgegriffen wurde auf das Verzeichnis jüdischer Autoren der Reichsstelle zur Förderung des deutschen Schrifttums, die man als bislang größte und gleichwohl fehlerhafte Sammlung von Informationen nutzte; zugleich verwendete man Nachschlagewerke wie die Große jüdische Nationalbiographie von Wininger, das von den NS-Akteuren selbst

54 Planung einer „Bibliographie des jüdischen Schrifttums in deutscher Sprache“ vom 15. April 1941. ADNBL I 612/0.

55 ADNBL I 612/0, Bl. 1. Entsprechend wurde als Bedarf für eine „Bibliographie des jüdischen Schrifttums für den Zeitraum von 1901-1940“ angefordert: „1 wiss. Kraft (T[arif]O[rdnung] A III); 3 geprüfte Kräfte des wissenschaftlichen Bibliotheksdienstes (T[arif]O[rdnung] A VIb), 2 Schreibmaschinenkräfte" sowie Materialkosten.

56 Ministervorlage von Leiter der Abteilung S (Schrifttum) vom 24. Juni 1941. ADNBL 612/0, Bl. 5. 57 So der Bericht von Bibliotheksrat Hans Ruppert über eine Dienstreise nach Berlin in Sachen der Bibliographie des jüdischen Schrifttums am 19. und 20. Februar 1942. ADNBL I 612/0, Bl. 13. 
als unwissenschaftlich erkannte antisemitische Personen- und Sachlexikon Sigilla Veri sowie das 1938 erschienene Philo-Lexikon, das schon die Trennung von Voll- und Halbjuden, weiteren Mischlingen und jüdisch Versippten praktizierte. Dennoch wurde die Arbeit fortgeführt und weiterhin vom RMVP alimentiert. ${ }^{58}$

Wichtiges Recherche-Instrument waren standardisierte Anfrage-Karten, die von der BjS-Stelle an Einwohner-Meldeämter, Universitätsverwaltungen und Verlage geschickt wurden und folgenden Wortlaut hatten:

Die Deutsche Bücherei ist vom Reichsministerium für Volksaufklärung und Propaganda mit der Bearbeitung eines Verzeichnisses der jüdischen Schriftsteller in deutscher Sprache 1901-1940 beauftragt. Hierfür bitte ich, soweit möglich unter Angabe der Quellen, um gefällige Mitteilung auf der angebogenen Karte über Abstammungsverhältnisse und Bekenntnis von Max Hermann (-Neisse), geboren dort am 25. 5. 1886 sowie seiner Eltern, wenn möglichst mit Geburtsort und Geburtsdatum. Die Deutsche Bücherei ist eine dem Reichsministerium für Volksaufklärung und Propaganda unterstehende Anstalt des öffentlichen Rechts. Es wird deshalb um gefällige gebührenfreie Erledigung gebeten. ${ }^{59}$

Die beigelegten Antwort-Postkarten wurden von Einwohner-Meldeämtern, Universitätsverwaltungen und Verlagen ausgefüllt und zurückgesandt; in Leipzig wurden diese Informationen aufgenommen und in die Karteikarten eingetragen. Auf dieser Basis erteilte man wiederum Auskünfte; so etwa an das Reichspropagandaministerium, das sich hier am 2. April 1943 in Gestalt des WissenschaftsReferenten Günther Lutz erkundigte, „ob die Vermutung, dass Prof. Erich Auerbach, Marburg, jüdischer Abstammung ist zutrifft“. ${ }^{60}$ - Zugleich bat das RMVP um Prüfung von Angeboten des deutschen Buchhandels, so etwa, wenn der Leiter des Referats Wissenschaft am 30. April 1943 zwei Kataloge des Verlages Max Niemeyer übersandte „mit der Bitte um Durchsicht nach jüdischen Autoren“:

Zum Teil sind sie bereits vom Verlag kenntlich gemacht worden. Es wird gebeten, jeweils einen kleinen Vermerk bei den betreffenden jüdischen Autoren an den Rand des Blattes zu zeichnen (z.B.: Jd., Halbjd., Vierteljd., jüd. versippt.) Gleichzeitig bedeuten die in den Katalogen aufgeführten jüdischen Autoren sicher eine Erweiterung ihrer jüdischen Autorenkartei. ${ }^{61}$

Dabei muss die Arbeitsstelle an der DB auch böswillige Gerüchte und Verleumdungen aus dem Weg räumen, so etwa die Aussage, „August Sauer, ehemals Prof. für neuere deutsche Literatur an der Universität Prag, soll Jude

58 Vgl. das Schreiben von Wilhelm Haegert vom 5. Februar 1943. ADNBL 612/0, Bl. 26a.

59 ADNBL 612/1, Bl. 14.

60 Günther Lutz an Hans Ruppert. Karte vom 2. April 1943. ADNBL 612/1, Bl. 19.

61 Günther Lutz an Hans Ruppert. Einschreiben vom 30. April 1943. ADNBL 612/1, Bl. 36. 
sein. Im Prager Hochschulstreik, der wegen des jüdischen Rektors Steinherz ausgebrochen war, hat der Genannte die jüdischen Interessen, wie angegeben wurde, vertreten."62 - In seiner Antwort teilt der Dekan der Philosophischen Fakultät der Deutschen Karls-Universität in Prag mit, „dass der Präsident der Deutschen Akademie in Prag Prof. Dr. Grosser Einblick in den Ahnenpass des Prof. Dr. August Sauer genommen hat, über dessen arische Abkunft keine Zweifel bestehen.“63

Die Arbeit wird im März 1944 eingestellt. Doch noch am 14. Juli 1944 erklärt DB-Generaldirektor Heinrich Uhlendahl gegenüber dem RMVP, man sei bereit, „die Arbeit fortzuführen, sofern das Ministerium diese unter den gegenwärtigen Kriegsverhältnissen für notwendig erachtet und vom Arbeitsamt die hierfür erforderlichen Arbeitskräfte $\mathrm{zu}$ erlangen sind““.64 Wie erwähnt gehört $\mathrm{zu}$ den letzten Nutzerinnen dieser Bibliographie des jüdischen Schrifttums Elisabeth Frenzel, die im ,Amt Rosenberg، für die Überwachung der deutschen Theaterliteratur zuständig ist; am 27. Oktober 1944 bedankt sie sich bei Bibliotheksrat Hans Ruppert und erklärt: „Durch die Einsichtnahme in Ihr Material bin ich doch in vielen Fällen weiter gekommen und hoffe, daß die endgültigen Ermittlungen auf Grund dieses Materials nun nicht mehr auf zu große Schwierigkeiten stoßen.“"65

Ein materiales Ergebnis der bio-bibliographischen Recherchen an der DB stellt möglicherweise der von Sören Flachowsky im Bundesarchiv Berlin-Licherfelde gemachte Fund unter den Überlieferungen des Reichspropagandaministeriums dar. Das Karteikarten-Konvolut „Nichtarische Schriftsteller, A-E“ (BArch R 55/21600) erfasst Parameter von Literaturproduzenten und macht diese - genau wie die an der DB erstellte ,Judenbibliographie“ - sichtbar:

62 Unleserlich [W. Emrich?] im Auftrag von Dr. Günther Lutz an Ruppert. Brief vom 30.3.1943. ADNBL 612/1, Bl. 39.

63 Dekan der Philosophischen Fakultät der Deutschen Karls-Universität in Prag an die Deutsche Bücherei. Brief vom 17. Mai 1943. ADNBL 612/1, Bl. 41.

64 ADNBL 612/0, Bl. 42.

65 Elisabeth Frenzel an Hans Ruppert. Brief vom 27.10.1944. ADNBL 612/1, Bl. 157. 


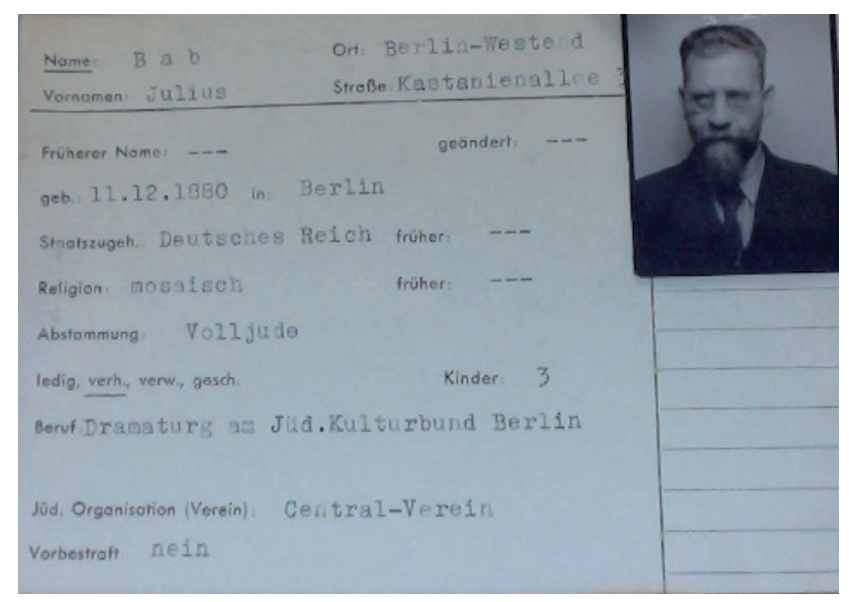

Abb. 2: Registraturkarte Julius Bab in der Kartothek Nichtarische Schriftsteller, $A-E$, BArch R 55/21600. ${ }^{66}$

Erfassung und Visibilisierung der als ,weltanschauliche Gegner' geltenden Autoren sind eine Aufgabe des RMVP-Erkundungsreferats. Andere Aufgaben der Schrifttumsbeobachter im RMVP bestehen in ,Revisionsverfahren ' und also in inhaltsbezogenen Kontrollen aktuell erscheinender Bücher. Hatte man die Bücher zu diesem Zweck bisher von der DB an das Ministerium geschickt, besteht eine Aufgabe des Erkundungsreferates nun darin, die nachhinkende Indizierungspraxis gleichsam ,vor Ort‘ auf Vordermann zu bringen. Schon 1937 hatte Karl Heinz Hederich, kurzzeitig der Amtschef der Abteilung VIII, die in seinen Augen mangelhafte Schrifttumskontrolle des Reichspropagandaministeriums - die durch Konkurrenten wie das Amt Rosenberg noch erschwert wurde - kritisiert und eine Umstrukturierung der Abteilung angeregt. Dabei sollte die ministerielle Zusammenarbeit mit der DB intensiviert und dort eine Dependance der Schrifttumsabteilung errichtet werden. Mit anderen Worten: Das von Wilhelm Emrich bekleidete Erkundungsreferat bildet einen direkten Link zwischen DB und RMVP, um auf kurzem Amtsweg über Zulassung oder Indizierung des eingehenden Schrifttums zu entscheiden.

66 Die Kartothek Nichtarische Schriftsteller, $A-E$ befindet sich in der Überlieferung des RMVP im Bundesarchiv Berlin-Lichterfelde (BArch R 55/21600) und kann möglicherweise als Ergebnis der in der DB Leipzig von 1941 bis 1944 erstellten Bibliographie des jüdischen Schrifttums in deutscher Sprache angesehen werden, an der Emrich als „Erkundungsreferent“ beteiligt war; siehe dazu Dienstleistungszeugnis für Wilhelm Emrich vom 12. April 1944. Für die freundliche Mitteilung dieses Fundes sowie für zahlreiche Hinweise danke ich Sören Flachowsky. 
Folgt man dieser Funktionsbestimmung lassen sich weitere Resultate der Tätigkeit von Wilhelm Emrich als ,Erkundungsreferent" des Reichspropagandaministeriums an der DB entdecken: Es sind Buchverbote mit Begründungen, die bei der Überwachung des an der DB eingehenden Schrifttums formuliert und in den als ,streng vertraulich' klassifizierten Periodika des Reichspropagandaministeriums Zeitschriften-Dienst und Deutscher Wochendienst veröffentlicht werden. Signifikant ist die hohe Zahl von Büchern aus der Schweiz, die verboten bzw. in die Liste 1 des schädlichen und unerwünschten Schrifttums eingereiht werden.

Auch wenn die Autorschaft dieser Verbotsbegründungen noch nicht geklärt ist, lassen sich exemplarische Zeugnisse für Indizierungspraxis und gelieferte Legitimationen herausgreifen. So wird am 26. Juni 1942 Philipp de Vards Kampfschrift Antisemitismus heilbar! Warum und wie aus dem Zürcher Verlag Zum Gutenberg verboten - mit der Begründung: „Das Buch von de Vard stellt ein Pamphlet gegen die deutsche Rassenlehre dar. Das Buch des Führers ,Mein Kampf' wird im gegnerischen Sinne ausgewertet. Dem Führer werden Aggressionsabsichten auf die ganze Welt untergeschoben." ${ }^{667}$ Am 3. Juli 1942 wird in die Liste 1 des schädlichen und unerwünschten Schrifttums Emil J. Walters Übersichtsdarstellung Europäischer Sozialismus. Rückblick und Ausblick aus dem Verlag Der Aufbruch (Zürich/New York 1941) eingereiht - mit der Begründung: „Die Darstellung des jetzigen Krieges erfolgt ausschließlich aus marxistisch-klassenkämpferischen Gesichtspunkten. Als Wunschziel nach dem Krieg wird ein ,europäischer Sozialismus‘, d.h. eine ,öffentliche demokratische Selbstverwaltung der Wirtschaft auf genossenschaftlicher Grundlage;, also ein schweizerischer Idealzustand, vorgeschlagen. “68 Welche unterschiedlichen Anforderungen die Schrifttumsüberwacher zu bewältigen hatten, zeigt sich in der Verbotsliste, die im Deutschen Wochendienst vom 31. Juli 1942 erscheint:

In die Liste 1 des schädlichen und unerwünschten Schrifttums wurden eingereiht:

1. Ernst Schürch: ,Bemerkungen zum Tage‘. Verlag Paul Haupt. Bern 1942

Der Verfasser steht auf dem Boden einer individualistisch-demokratischen Weltanschauung. Er lehnt die Politik der Achsenmächte ab. Seine Warnung an die Schweizer, sich unter dem Druck der deutschen Siege nicht zu sehr auf die Seite der Achsenmächte zu stellen, kommt einer Parteinahme für die Feindmächte gleich.

67 Verbotene Bücher (Nr. 7075). In: Deutscher Wochendienst vom 26. Juni 1942. Zugleich wird ein Verbot sämtlicher Schriften des Verlages Zum Gutenberg ausgesprochen.

68 Verbotene Bücher. In: Deutscher Wochendienst vom 3. Juli 1942. Zugleich wird ein Verbot sämtlicher Schriften des Verlages Der Aufbruch, Zürich/New York ausgesprochen. Verboten werden an diesem Tag ebenfalls die Schriften von Wilhelm Vischer: Die Judenfrage, eine entscheidende Frage für die Kirche. Basel: Verlag Evangelische Buchhandlung 1942; Kurt Fueter: Gott sitzt im Regimente! Zollikon/Zürich: Evangelischer Verlag 1942. 
2. Margarete Mueller-Senftenberg: ,Götzendämmerung in Wissenschaft und Religion‘. Robert Kaemmerer Verlag, Berlin o.J.

Das Buch ist eine Mischung aus theosophischen, psychoanalytischen, okkultistischen und christlichen Gedankengängen. Es wendet sich gegen den Antisemitismus, stellt die SowjetUnion als Vorbild einer ins Geistige übertragenen Räterepublik für Deutschland hin und steht in starker Abhängigkeit von amerikanischen Autoren.

3. W. Brandt: ,Norge fortsätter kampen‘. Verlag Albert Bonniers, Stockholm 1941

Die Schrift ist als Kampfschrift gegen die deutschen Maßnahmen in Norwegen verfaßt und ruft zum offenen Widerstand gegen das Deutsche Reich und die mit ihm sympathisierenden norwegischen Kreise auf. ${ }^{69}$

Nur spekulieren lässt sich über die Hintergründe und die Motive der hier ausgesprochenen Buchverbote. Was wusste der Zensor beispielsweise über die Biographie des Theologen und Pfarrers Kuno Fiedler, dessen Buch Schrift und Schriftgelehrte. Eine kleine Rüstkammer er im Juni 1942 in die Liste 1 des schädlichen und unerwünschten Schrifttums einreihte? Immerhin erkannte er in dem verbotenen Buch „ein Christentum in liberalistisch-universalistischer Richtung“, das „dem nationalsozialistischen Weltbild diametral entgegen" gestellt sei. Doch ahnte er, dass Kuno Fiedler ein Freund des Schriftstellers Thomas Mann war, den Emrich in seiner Frankfurter Zeit bewundert und umworben hatte? Hatte er eine Ahnung von den Verbindungen zwischen dem Großschriftsteller und dem evangelischen Theologen, der am 23. Oktober 1918 die Haustaufe von Thomas Manns jüngster Tochter Elisabeth vorgenommen hatte (und im hexametrischen Gesang vom Kindchen als „verordneter Jüngling“ sanft verspottet worden war)? Kannte er die Geschichte von Fiedlers Flucht aus dem Würzburger Zuchthaus 1935 und seiner nachfolgenden Aufnahme in der Schweiz, die durch nachhaltige Unterstützung von Thomas Mann möglich geworden war? - Aufschlussreicherweise hatte Fiedler schon im Jahr der NS-Machtübernahme die Anpassungsleistungen der deutschen Mandarine beobachtet: In einem Brief an Thomas Mann vom 6. August 1933 berichtete er, „dass so ziemlich das ganze Volk bei uns ,mitmacht‘. Auch die Gebildeten. Der Fall Ihres Freundes Bertram ist nicht vereinzelt. Jeder, der irgendwo ein Pöstchen hat, legt jetzt die schärfste Linse ein, um an dem neuen System ,Vorzüge' zu entdecken." ${ }^{\text {”o }}$

69 Verbotene Bücher. In: Deutscher Wochendienst vom 31. Juli 1942.

70 Zitiert nach Thomas Sprecher: Pfarrer Kuno Fiedler - Weggefährte und Korrespondent Thomas Manns. In: Bündner Jahrbuch. Neue Folge 41 (1999), S. 94-104, hier S. 98. Thomas Mann kommentiert den Bericht Fiedlers im Tagebucheintrag vom 10. August 1933: „Fiedler, der von rückgratlosem ,Mitmachen“ in Deutschland erzählt, den Wendungen, mit denen man es entschuldigt: ,Erschütterung‘, ,Geistige Strukturveränderung, für die die alten Massstäbe nicht gelten'“"Vgl. auch die Einträge vom 26. September 1933, 14. Oktober 1933 und 17. September 1934: 
Wenig wahrscheinlich ist, dass Wilhelm Emrich in diesen Tagen und Wochen der Tätigkeit an der Deutschen Bücherei die BBC-Radioansprachen Deutsche Hörer verfolgte, mit denen sich Thomas Mann an die Bevölkerung in NaziDeutschland wandte und die gleichfalls auf Berichten von Kuno Fiedler beruhten. Denn auch wenn er in Briefen aus dieser Zeit über „Willensfreiheit“ und eskapistische Fluchten aus der Gegenwart schwadronierte, ${ }^{71}$ verrichtete er seine Arbeit im Dienst des Systems.

Am 31. Januar 1964 hält Wilhelm Emrich, der zwischen 1959 und 1974 an der Freien Universität Berlin lehren und mehrere Generationen von Studierenden prägen sollte, im Rahmen der Universitätstage einen Vortrag unter dem Titel „Kann Dichtung verboten werden?“72 Anlass sind publizistisch diskutierte Probleme mit provokativ freizügiger Gegenwartsliteratur. Die nun vorgebrachten Überlegungen des Mannes, der als Zensor im Goebbels-Ministerium gearbeitet hatte, werfen weiteres Licht auf die Dienstleistungsgeschichte der deutschen Philologie. Doch dieses Kapitel kann hier leider nicht mehr dargestellt werden.

Thomas Mann: Tagebücher. Hg. von Peter de Mendelssohn bzw. Inge Jens. Frankfurt/M.: S. Fischer 1979-1995.

71 Wilhelm Emrich an Lenna Emrich. Brief vom 07. Juni 1942. Deutsches Literaturarchiv Marbach, Nachlass Emrich: „Ich glaube auch an die Willensfreiheit. ich glaube daran auch gegen alle Vernunft und alle leidige Wirklichkeit.“ Am 12. Juni 1942: „Ich glaube auch, daß wir unsere Gegenwart vergessen können und von einem größeren Strom der Zeit getragen werden.“

72 Veröffentlicht im Sammelband von Werner Maihofer (Hg.): Gesellschaftliche Wirklichkeit im 20. Jahrhundert und Strafrechtsreform. Berlin: Walter de Gruyter 1964, S. 159-172. 